\title{
Correlations between the stellar, planetary, and debris components of exoplanet systems observed by Herschel ${ }^{\star}$
}

\author{
J. P. Marshall ${ }^{1,2}$, A. Moro-Martín ${ }^{3,4}$, C. Eiroa ${ }^{1}$, G. Kennedy 5 , A. Mora ${ }^{6}$, B. Sibthorpe ${ }^{7}$, J.-F. Lestrade ${ }^{8}$, \\ J. Maldonado ${ }^{1,9}$, J. Sanz-Forcada ${ }^{10}$, M. C. Wyatt ${ }^{5}$, B. Matthews ${ }^{11,12}$, J. Horner ${ }^{2,13,14}$, B. Montesinos ${ }^{10}$, G. Bryden ${ }^{15}$, \\ C. del Burgo ${ }^{16}$, J. S. Greaves ${ }^{17}$, R. J. Ivison ${ }^{18,19}$, G. Meeus ${ }^{1}$, G. Olofsson ${ }^{20}$, G. L. Pilbratt ${ }^{21}$, and G. J. White ${ }^{22,23}$ \\ (Affiliations can be found after the references)
}

Received 15 November 2013 / Accepted 6 March 2014

\begin{abstract}
Context. Stars form surrounded by gas- and dust-rich protoplanetary discs. Generally, these discs dissipate over a few (3-10) Myr, leaving a faint tenuous debris disc composed of second-generation dust produced by the attrition of larger bodies formed in the protoplanetary disc. Giant planets detected in radial velocity and transit surveys of main-sequence stars also form within the protoplanetary disc, whilst super-Earths now detectable may form once the gas has dissipated. Our own solar system, with its eight planets and two debris belts, is a prime example of an end state of this process.

Aims. The Herschel DEBRIS, DUNES, and GT programmes observed 37 exoplanet host stars within $25 \mathrm{pc}$ at 70, 100, and $160 \mu \mathrm{m}$ with the sensitivity to detect far-infrared excess emission at flux density levels only an order of magnitude greater than that of the solar system's Edgeworth-Kuiper belt. Here we present an analysis of that sample, using it to more accurately determine the (possible) level of dust emission from these exoplanet host stars and thereafter determine the links between the various components of these exoplanetary systems through statistical analysis.

Methods. We have fitted the flux densities measured from recent Herschel observations with a simple two parameter $\left(T_{d}, L_{\mathrm{IR}} / L_{\star}\right)$ black-body model (or to the $3 \sigma$ upper limits at $100 \mu \mathrm{m}$ ). From this uniform approach we calculated the fractional luminosity, radial extent and dust temperature. We then plotted the calculated dust luminosity or upper limits against the stellar properties, e.g. effective temperature, metallicity, and age, and identified correlations between these parameters.

Results. A total of eleven debris discs are identified around the 37 stars in the sample. An incidence of ten cool debris discs around the Sun-like exoplanet host stars $(29 \pm 9 \%)$ is consistent with the detection rate found by DUNES $(20.2 \pm 2.0 \%)$. For the debris disc systems, the dust temperatures range from 20 to $80 \mathrm{~K}$, and fractional luminosities $\left(L_{\mathrm{IR}} / L_{\star}\right)$ between $2.4 \times 10^{-6}$ and $4.1 \times 10^{-4}$. In the case of non-detections, we calculated typical $3 \sigma$ upper limits to the dust fractional luminosities of a few $\times 10^{-6}$.

Conclusions. We recover the previously identified correlation between stellar metallicity and hot-Jupiter planets in our data set. We find a correlation between the increased presence of dust, lower planet masses, and lower stellar metallicities. This confirms the recently identified correlation between cold debris discs and low-mass planets in the context of planet formation by core accretion.
\end{abstract}

Key words. infrared: stars - infrared: planetary systems - circumstellar matter - planet-disk interactions

\section{Introduction}

Circumstellar debris discs around main-sequence stars are composed of second-generation dust produced by the attrition of larger bodies (Backman \& Paresce 1993), which are remnants of primordial protoplanetary discs (Hernández et al. 2007). Debris discs can be detected and analysed based on their thermal infrared emission from the constituent dust particles. Around $16.4_{-2.9}^{+2.8} \%$ of main-sequence Sun-like stars have evidence of circumstellar dust emission at $70 \mu \mathrm{m}$ with Spitzer (Trilling et al. 2008). From observations of FGK stars by the Herschel ${ }^{1}$ DUNES survey an incidence of $20.2 \pm 2.0 \%$ was measured (Eiroa et al. 2013), whereas the DEBRIS survey measures an incidence of $16.5 \pm 2.5 \%$ (Sibthorpe et al., in prep.).

Many circumstellar discs around Sun-like stars are seen to have two temperature components, which has been interpreted

\footnotetext{
* Tables 2-4 are available in electronic form at http: //www . aanda. org

1 Herschel is an ESA space observatory with science instruments provided by European-led Principal Investigator consortia and with important participation from NASA.
}

as arising from two distinct belts at different stellocentric radii (Chen et al. 2009; Morales et al. 2011). The cool discs are more commonly seen and analogous to the Edgeworth-Kuiper belt (EKB) in our own solar system (Greaves \& Wyatt 2010; Vitense et al. 2012). The EKB's existence has been inferred from the detection of over a thousand trans-Neptunian objects ${ }^{2}$, through ground-based surveys and in situ dust measurement from Voyager 1 and 2 (Gurnett et al. 1997) and New Horizons (Poppe et al. 2010; Han et al. 2011), although direct observation of the dust emission from the EKB is confounded by the bright foreground thermal emission from the zodiacal dust in the inner solar system (Backman et al. 1995). The less commonly seen warm debris disc asteroid-belt analogues, which are more difficult to observe around other stars due to the larger flux density contribution from the stellar photosphere at mid-infrared wavelengths compared with the dust excess, have been detected around $\sim 2 \%$ of Sun-like stars (3/7 FGK stars with $24 \mu \mathrm{m}$ excess and $T_{\text {dust }}>100 \mathrm{~K}$ from a sample of 184, Trilling et al. 2008).

\footnotetext{
1258 as of 29th October 2013, see:

http://www .minorplanetcenter. net/iau/lists/TNOs.html
} 
Exoplanets $^{3}$ around Sun-like stars have been identified through radial velocity (e.g. Marcy \& Butler 2000; Tinney et al. 2001; Mayor \& Queloz 2012) or transit surveys e.g. CoRoT (Auvergne et al. 2009), WASP (Pollacco et al. 2006), HAT (Bakos et al. 2002) and Kepler (Borucki et al. 2011). See Perryman (2011) for a summary of exoplanet detection techniques. The majority of all exoplanet searches have taken place at optical wavelengths, with a sample focus on mature, Sun-like stars as the most suitable candidates for the radial velocity detection technique. A stars are avoided as their atmospheres lack the narrow lines necessary for radial velocity detections through accurate Doppler measurements, but these stars are prime candidates for direct imaging surveys, resulting in the detection of several exoplanet systems around debris disc host stars, e.g. Fomalhaut (Kalas et al. 2008), HR 8799 (Marois et al. 2008), $\beta$ Pic (Lagrange et al. 2010) and HD 95086 (Rameau et al. 2013). M stars have likewise been avoided because they exhibit high levels of stellar variability and because of their emission peaks in the near-infrared, which render them noisy and faint, although great efforts have been made to overcome these problems because of their sheer number and potential to yield low-mass planets through either transit or radial velocity detections (e.g. Reiners et al. 2010; Rodler et al. 2011; Giacobbe et al. 2012; Anglada-Escudé \& Tuomi 2012). This has led to unavoidable bias in the types of stars around which exoplanets have been studied. Comparative analysis or aggregation of results from radial velocity surveys is further complicated by both the differences in sensitivity and the variable baseline of observations for the stellar samples, although broad conclusions, for instance on the absence of Jupiter analogues around most nearby stars, may be drawn (Cumming et al. 1999; Fischer et al. 2014). Recent results from an analysis of microlensing surveys suggest that almost all stars may have one or more exoplanets, with lowmass planets being much more common than Jupiter-mass ones (Cassan et al. 2012). On the other hand, long-term monitoring from the ground has constrained the likelihood of Jovian planets on long orbits (3-6 AU) to $<30 \%$ of the stellar systems surveyed (Wittenmyer et al. 2011), suggesting that exoplanetary systems very like our own may be rare, a result supported by recent direct imaging searches (Janson et al. 2013).

Given that planets are the end state of the agglomeration of smaller bodies from dust to planetesimals (neglecting as a detail the capture of an envelope from the protoplanetary disc for gas giants), and debris discs are the result of collisional grinding of these planetesimals into dust, one might expect the presence of planets and debris discs to be correlated. This expectation is strengthened by the direct imaging of several exoplanet systems around debris disc host stars, as previously noted, and indirectly by the structural features observed in many debris discs (warps, off-sets, asymmetries). These features have generally been thought to arise from the gravitational perturbation of exoplanets (see reviews by Wyatt 2008; Krivov 2010; Moro-Martin 2013), although remnant gas may offer an alternative explantation in some cases (e.g. Lyra \& Kuchner 2013). Evidence of disc structures, particularly in the sub-mm, has sometimes weakened upon closer scrutiny, for example the SCUBA detected "blob" in Vega's disc (Holland et al. 1998; Wyatt 2003; Piétu et al. 2011; Hughes et al. 2012) or the clumps in the disc of HD 107146 (Corder et al. 2009; Hughes et al. 2011), requiring that caution be exercised when attributing these structures to unseen planetary bodies. Until recently, no clear correlation had yet been

\footnotetext{
Databases of exoplanet properties are maintained at http:// exoplanet. eu and http://exoplanets.org
}

established between the presence of debris discs and the presence of planets (Greaves et al. 2006; Moro-Martín et al. 2007; Bryden et al. 2009; Kóspál et al. 2009), and larger scale direct imaging surveys have found little evidence of massive planets around debris disc host stars (Wahhaj et al. 2013; Janson et al. 2013). However, new analysis by Maldonado et al. (2012) identified a trend between the presence of a debris disc and cool Jupiter exoplanet around a star, whilst Wyatt et al. (2012) identified a possible trend between cool dust and low-mass planet host stars.

Direct measurement of the spatial distribution of debris in other stellar systems will reveal whether our own EKB is common or unusual. The EKB and its interaction with the outer planets is thought to have played a significant role in the development of life on Earth, having supplied a significant fraction of the impactors thought to have been involved in the Late Heavy Bombardment (Gomes et al. 2005), dictated the development of the terrestrial planets (Walsh et al. 2011), and continues to provide bodies for the short-period comet population through interaction with Jupiter (Horner \& Jones 2009). It may also have been involved in the hydration of Earth (Morbidelli et al. 2000), a topic that is still widely debated (Raymond et al. 2004, 2006, 2007; O'Brien et al. 2006; Horner et al. 2009; Bond et al. 2010; Horner \& Jones 2010; Izidoro et al. 2013). As such, the nature of exo-EKBs may play a vital role in the determination of the habitability of their host systems (e.g. Horner \& Jones 2010), and it is therefore vital that we are able to judge whether the solar system's architecture and EKB are the norm, or unusual.

The Herschel (Pilbratt et al. 2010) guaranteed time (GT) debris disc programme and the open time key programmes Disc Emission from Bias-free Reconnaissance Infrared Survey (DEBRIS ${ }^{4}$; Matthews et al. 2010) and DUst around NEarby Stars (DUNES ${ }^{5}$; Eiroa et al. 2010, 2013) have observed nearby Sun-like stars using the Photodetector Array Camera and Spectrometer instrument (PACS; Poglitsch et al. 2010) at farinfrared wavelengths searching for excess emission caused by circumstellar dust discs analogous to the solar system's EKB (Vitense et al. 2012). In this work, we have examined all of the stars from the DEBRIS, DUNES and GT programmes that are currently believed to host exoplanets.

In Sect. 2, we present the observations used in this work, the data reduction process, and the stellar physical parameters to be compared with the dust emission. In Sect. 3, a summary of the assumptions used to fit models to the new Herschel photometry are explained and the calculated disc temperatures and masses are shown. In Sect. 4, a comparison of the observed disc fractional luminosities (or $3 \sigma$ upper limits in the case of nondetections) from Sect. 3 and the stellar properties from Sect. 2 is presented. Finally, in Sect. 5, we present our conclusions and recapitulate our findings.

\section{Observations and data reduction}

The observations used in this work have been taken from the DEBRIS and DUNES open time key programmes and the debris disc guaranteed time programme. The sample comprises the 14 stars from DEBRIS and 21 stars from DUNES that are mainsequence stars within 25 pc of the Sun with radial-velocitydetected exoplanets. We also added $\tau$ Ceti and $\epsilon$ Eridani from the guaranteed time key programme. We included both $\alpha$ Centauri B and $\epsilon$ Eridani in the sample although there is some doubt about the existence of their respective planets: $\alpha$ Centauri B;

\footnotetext{
4 http://debris.astrosci.ca

5 http://www .mpia-hd.mpg.de/DUNES/
} 
Dumusque et al. (2012); Hatzes (2013), and $\epsilon$ Eridani; Hatzes et al. (2000); Moran et al. (2004); Zechmeister et al. (2013). There are no A stars that match the criteria for inclusion; although Fomalhaut and $\beta$ Pictoris both lie within the volume, their planets are directly imaged. Likewise, around G stars, GJ 504 hosts a cold Jovian planet (Kuzuhara et al. 2013) and HN Peg has a borderline brown dwarf/Jovian companion (Faherty et al. 2010). The range of spectral types represented in the sample is therefore F to M. Several exoplanet host stars have not been included, for example HD 136352, HD 147513, and HD 190360 (Wyatt et al. 2012), even though they lie within the volume explored by DEBRIS and DUNES, because they all lie towards regions of bright far-infrared background contamination from Galactic emission and were therefore omitted from observation by the Herschel programmes.

Herschel PACS 70/160 and/or 100/160 scan map observations were taken of all stars except $51 \mathrm{Peg}$, which was observed in chop-nod mode with the 100/160 channel combination during the science demonstration phase (SDP). The observation parameters for the DEBRIS and DUNES targets were slightly different: for DEBRIS targets, each scan map consisted of two repetitions of eight scan legs of $3^{\prime}$ length, with a $4^{\prime \prime}$ separation between legs, taken at the medium slew speed (20" per second), whereas DUNES targets had the same parameters, but used ten legs per scan map and the number of repetitions was dictated by the requirement to detect the stellar photosphere at $100 \mu \mathrm{m}$ with a signal-to-noise ratio $(S / N) \geq 5$. The PACS 100/160 scan map observations of $\mathrm{q}^{1}$ Eri were observed as part of the calibration effort during the SDP phase using scans with 16 legs of 3.9' with 4 " separation between the legs, also at the medium slew speed. For the GT programme, the targets were observed twice, with each scan map consisting of 11 scan legs of 7.4' length, with a 38" separation between legs, at the medium slew speed. Each of the GT scans was repeated 11 times. In all cases, each target was observed at two array orientation angles $\left(70^{\circ}\right.$ and $110^{\circ}$ for DEBRIS and DUNES, $45^{\circ}$ and $135^{\circ}$ for GT), which were combined into a final mosaic to improve noise suppression and assist in the removal of instrumental artefacts and glitches. Several of the targets presented in this work have been observed by Herschel Spectral and Photometric Imaging REceiver (SPIRE; Griffin et al. 2010; Swinyard et al. 2010), but because of the sparse coverage of the sample with that instrument (4/37 targets) we decided to focus here on the PACS photometry to ensure consistency in the data set used for the sample analysis. A summary of all observations is presented in Table 1.

All observations were reduced interactively using version 8.1.0 of the Herschel Interactive Processing Environment (HIPE, Ott 2010) using PACS calibration version 32 and the standard scripts supplied with HIPE. Individual PACS scans were processed with a high-pass filter to remove background structure, using high-pass filter widths of 15 frames at $70 \mu \mathrm{m}, 20$ frames at $100 \mu \mathrm{m}$, and 25 frames at $160 \mu \mathrm{m}$, equivalent to spatial scales of $62^{\prime \prime}, 82^{\prime \prime}$, and $102^{\prime \prime}$. In the case of $\epsilon$ Eridani, with its much larger disc $\left(\sim 1^{\prime}\right.$ in diameter), a high-pass filter width of 50 frames, equivalent to $202^{\prime \prime}$, at both 70 and $160 \mu \mathrm{m}$ was adopted to avoid removal of disc flux by the filtering. For the filtering process, regions of the map where the pixel brightness exceeded a threshold defined as twice the standard deviation of the non-zero elements in the map were masked from the highpass filter task. The two individual scans of each target were mosaicked to reduce sky noise and suppress the striping due to detector scanning. Final image scales were $1^{\prime \prime}$ per pixel at $70 \mu \mathrm{m}$ and $100 \mu \mathrm{m}$ and $2^{\prime \prime}$ per pixel at $160 \mu \mathrm{m}$ compared with native
Table 1. Summary of Herschel PACS observations of the target stars.

\begin{tabular}{lrccr}
\hline \hline \multicolumn{1}{c}{ Target } & \multicolumn{1}{c}{ PACS } & Obs. IDs & OD $^{a}$ & \multicolumn{1}{c}{ OT } \\
& {$[\lambda \mu \mathrm{m}]$} & {$[1342 \ldots]$} & & \multicolumn{1}{c}{$[\mathrm{s}]$} \\
\hline HD 1237 & $100 / 160$ & $204272 / 73$ & 484 & 576 \\
HD 3651 & $70 / 160$ & $213242 / 43$ & 621 & 360 \\
& $100 / 160$ & $213244 / 45$ & 621 & 1080 \\
HD 4308 & $70 / 160$ & $212704 / 05$ & 612 & 180 \\
& $100 / 160$ & $212706 / 07$ & 612 & 1440 \\
$v$ And & $100 / 160$ & $223326 / 27$ & 777 & 360 \\
q ${ }^{1}$ Eri & $70 / 160$ & $212838 / 39$ & 614 & 360 \\
& $100 / 160$ & $187139 / 40$ & 191 & 768 \\
$\tau$ Ceti & $70 / 160$ & $213575 / 76$ & 628 & 5566 \\
GJ 86 & $70 / 160$ & $214175 / 76$ & 639 & 180 \\
& $100 / 160$ & $214177 / 78$ & 639 & 720 \\
$\iota$ Hor & $100 / 160$ & $214196 / 97$ & 640 & 576 \\
HD 19994 & $100 / 160$ & $216129 / 30$ & 661 & 1440 \\
HD 20794 & $100 / 160$ & $216456 / 57$ & 675 & 1220 \\
$\epsilon$ Eri & $70 / 160$ & $216123 / 24$ & 661 & 5566 \\
HD 33564 & $100 / 160$ & $219019 / 20$ & 705 & 720 \\
HD 39091 & $100 / 160$ & $205212 / 13$ & 502 & 576 \\
HD 40307 & $70 / 160$ & $203666 / 67$ & 471 & 180 \\
& $100 / 160$ & $203668 / 69$ & 471 & 1440 \\
HD 69830 & $100 / 160$ & $196125 / 26$ & 361 & 900 \\
55 Cnc & $100 / 160$ & $208504 / 05$ & 545 & 720 \\
47 UMa & $100 / 160$ & $198845 / 46$ & 403 & 360 \\
HD 99492 & $100 / 160$ & $212056 / 57$ & 582 & 576 \\
HD 102365 & $100 / 160$ & $202240 / 41$ & 450 & 576 \\
61 Vir & $100 / 160$ & $202551 / 52$ & 454 & 576 \\
70 Vir & $100 / 160$ & $213093 / 94$ & 615 & 1440 \\
$\tau$ Boo & $100 / 160$ & $213081 / 82$ & 615 & 360 \\
$\alpha$ Cen B & $100 / 160$ & $224848 / 49$ & 807 & 360 \\
GJ 581 & $100 / 160$ & $202568 / 69$ & 454 & 576 \\
$\rho$ CrB & $100 / 160$ & $215376 / 77$ & 662 & 1080 \\
14 Her & $100 / 160$ & $205996 / 97$ & 511 & 1440 \\
HD 154345 & $70 / 160$ & $193509 / 10$ & 324 & 1080 \\
& $100 / 160$ & $193511 / 12$ & 324 & 1440 \\
$\mu$ Ara & $100 / 160$ & $215572 / 73$ & 663 & 360 \\
HD 176051 & $100 / 160$ & $205038 / 39$ & 497 & 576 \\
16 Cyg B & $100 / 160$ & $198501 / 02$ & 400 & 576 \\
HD 189567 & $100 / 160$ & $208851 / 52$ & 547 & 576 \\
HD 192310 & $100 / 160$ & $208466 / 67$ & 545 & 576 \\
GJ 832 & $100 / 160$ & $208845 / 46$ & 547 & 576 \\
HD 210277 & $100 / 160$ & $211126 / 27$ & 579 & 1440 \\
GJ 876 & $100 / 160$ & $198521 / 22$ & 400 & 576 \\
51 Peg & $100 / 160$ & 187255 & 198 & 682 \\
HD 217107 & $100 / 160$ & $198515 / 16$ & 400 & 576 \\
\hline & & & &
\end{tabular}

Notes. ${ }^{(a)}$ Operational day; ${ }^{(b)}$ on-source integration time.

instrument pixel sizes of $3.2^{\prime \prime}$ at $70 \mu \mathrm{m}$ and $100 \mu \mathrm{m}$ and $6.4^{\prime \prime}$ at $160 \mu \mathrm{m}$.

Point source flux densities were measured with aperture radii of $4^{\prime \prime}, 5^{\prime \prime}$, and $8^{\prime \prime}$ at $70 \mu \mathrm{m}, 100 \mu \mathrm{m}$, and $160 \mu \mathrm{m}$ to maximize the $\mathrm{S} / \mathrm{N}$ of the source (Eiroa et al. 2013). The mean full width at half maximum (FWHM) of the PACS instrument is 5.61", $6.79^{\prime \prime}$ and $11.36^{\prime \prime}$ in the three bands. Extended sources, identified by comparison of a 2D Gaussian fit with the source profile to the PSF FWHM in each band, were measured with varying aperture radii depending on the disc extent. The sky background level and rms noise for each target were estimated from the mean and standard deviation of the total flux density in 25 boxes of dimensions $7 \times 7$ pixels at $70 \mu \mathrm{m}, 9 \times 9$ pixels at $100 \mu \mathrm{m}$, and $7 \times 7$ pixels at $160 \mu \mathrm{m}$, chosen to match the aperture size of point sources. The boxes were placed randomly around the central area of the mosaic within a region $30^{\prime \prime}$ to $60^{\prime \prime}$ from the source 
position, or $90^{\prime \prime}-120^{\prime \prime}$ in the case of $\epsilon$ Eri, to avoid the central source and edges of the maps where the noise increases due to the non-uniform map coverage.

The appropriate aperture corrections were applied to the flux densities based on the PACS aperture photometry calibration (factors of $0.476,0.513$, and 0.521 for point sources, respectively). Correction factors appropriate to the aperture radius based on the point source encircled energy fraction were applied to the extended sources, which, although only an approximation to the true correction, is widely used. A calibration uncertainty of 5\% was assumed for all three PACS bands (Balog et al. 2014). The flux densities presented in Table 2 have not been colour corrected.

The stellar photosphere contribution to the total flux density was calculated from a synthetic stellar atmosphere model interpolated from the PHOENIX/Gaia grid (Brott \& Hauschildt 2005). All stars in the sample match the criteria of $d<25 \mathrm{pc}$ and luminosity class V except for HD 217107, which is luminosity class IV. The stellar models were scaled to the combined optical, near-infrared, and WISE data, where the WISE bands were not saturated or showed evidence of excess emission, following Bertone et al. (2004). The stellar physical parameters are given in Table 3. For the DUNES observed targets, the stellar parameters were taken from Eiroa et al. (2013). For the DEBRIS observed targets, the stellar parameters were calculated from archival data using the same procedure as for the DUNES targets, see Eiroa et al. (2013) for details of the method (Maldonado, priv. comm.). Stellar distances were taken from the revised HIPPARCos catalogue (van Leeuwen 2007). Stellar ages were computed following Eq. (3) in Mamajek \& Hillenbrand (2008) for the Ca II based values, whilst the X-ray stellar age was calculated through the relation between X-ray emission and stellar age according to Sanz-Forcada et al. (2011), where most of the ages displayed in Table 3 were calculated. Newly calculated X-ray ages are provided for HD 19994, HD 20794, HD 33564, HD 40307, HD 69830, 70 Vir, GJ 581 and HD 192310 according to the method in Sanz-Forcada et al. (2011).

\section{Analysis}

A determination of the presence of excess emission from each star was made on the basis of the excess significance, or $\chi$ value. The significance was calculated using the PACS 100 and $160 \mu \mathrm{m}$ flux densities and uncertainties in the following manner:

$\chi_{\lambda}=\frac{\left(F_{\lambda, \text { obs }}-F_{\lambda, \text { pred }}\right)}{\sqrt{\sigma_{\lambda, \mathrm{obs}}^{2}+\sigma_{\lambda, \text { pred }}^{2}+\sigma_{\lambda, \mathrm{cal}}^{2}}}$,

where $F_{\lambda \text {,obs }}$ and $F_{\lambda \text {,pred }}$ are the observed and predicted (photosphere) flux densities at the wavelength under consideration and the uncertainty is the quadratic sum of the uncertainties of the observation $\sigma_{\lambda \text {,obs }}$, the stellar photosphere model $\sigma_{\lambda \text {,pred }}$, and calibration $\sigma_{\lambda \text {,cal }}$.

The stars in the sample with $\chi_{\lambda}>3$ at either (or both) PACS wavelength(s) were classed as having significant excess emission. The disc fractional luminosity was calculated by fitting a black-body emission model parameterised by the dust temperature, $T_{\mathrm{d}}$, and fractional luminosity, $L_{\mathrm{IR}} / L_{\star}$, to the error-weighted Spitzer MIPS $70 \mu \mathrm{m}$ and Herschel PACS photometry.

For non-excess sources, the dust temperature was assumed to be $37 \mathrm{~K}$, and a $3 \sigma$ upper limit for the fractional luminosity was then calculated. Adopting a dust temperature of $37 \mathrm{~K}$ for calculation provides a strict minimum for the dust fractional luminosity of the non-excess stars based on the observed $100 \mu$ m flux density. The upper limits calculated here (presented in Table 2) are therefore consistent with the typical dust temperature we would expect to observe for debris discs around Sun-like stars.

Although crude, this model allows a consistent and uniform calculation of the dust properties from the observed flux densities, which is necessary for the statistical approach to identify trends in the data without getting distracted by the intricate details of individual sources, such as extended emission or material composition.

We note that for disc radii it is well known that an assumption of black-body emission can underestimate the radial distance of the dust from the star by a factor of 1-2.5 (for A stars, Booth et al. 2013) or a factor of up to 4 around $G$ and $M$ stars, for instance 61 Vir (Wyatt et al. 2012), HD 207129 (Marshall et al. 2011; Löhne et al. 2012), and GJ 581 (Lestrade et al. 2012). We derived radial locations for the dust from the assumption of thermal equilibrium between the dust grains and the incident radiation as a minimum possible orbital distance for the emitting dust in these systems (Backman \& Paresce 1993).

We calculated values for the basic physical parameters of the discs, which are presented in Table 2 (dust fractional luminosity, dust temperature, and dust radius) from the black-body fit to the spectral energy distribution and the assumption of black-body absorption and emission for the dust grains. We used the disc fractional luminosity for comparison with the stellar and exoplanet parameters, breaking down the observed sample of exoplanet host stars into three subsamples (low-mass planets, cool Jupiters, and hot Jupiters) to check for evidence of the trends identified in recent articles (e.g. Maldonado et al. 2012; Wyatt et al. 2012).

\section{Results}

We have detected far-infrared excess emission with $\chi>3$ from ten of the 37 systems in this sample. HD 69830 is known to have a warm debris disc (Beichman et al. 2005), bringing the total to 11 systems that have both a debris disc and exoplanet(s). The histogram presented in Fig. 1 illustrates the distribution of the measured (non-)excesses at 100 and $160 \mu \mathrm{m}$, with a long tail toward higher significances, as expected. Fitting Gaussian profiles to both distributions reveals that at $100 \mu \mathrm{m}$ the distribution is peaked at $\chi_{100}=-0.5$ with $\sigma_{100}=1.25$, whilst at $160 \mu \mathrm{m}$ the distribution peaks at $\chi_{160}=0.0$ with $\sigma_{160}=1.0$. The peak position and width of the Gaussian are measures of the goodness of our stellar photosphere and uncertainty estimates, respectively. We expect (assuming normally distributed uncertainties) that both $\chi$ distributions would peak at $\chi=0$ with $\sigma=1$. The discrepancy at $100 \mu \mathrm{m}$ might be ascribed to a systematic underestimation of the errors, which is hidden at $160 \mu \mathrm{m}$ by the larger uncertainties, but this same trend is seen in the larger DUNES sample of 133 stars (Eiroa et al. 2013). A physical explanation of the shift of the peak to $\chi_{100}<0$, implying a deficit in the measured flux density compared with the Rayleigh-Jeans extrapolation of the stellar photosphere model from $50 \mu \mathrm{m}$, might be found in the fact that the photosphere models do not take into account the decrease in brightness temperature in the higher layers of the stellar photosphere of Sun-like stars. This decrease in brightness temperature has the effect of reducing the observed flux density below that expected from an extrapolated fit to shorter wavelength measurements, with the greatest effect around $150 \mu \mathrm{m}$ (Eddy et al. 1969; Avrett 2003; Liseau et al. 2013). The magnitude of the deficit is $\sim 20 \%$, and similar to the magnitude of the measured uncertainty at $100 \mu \mathrm{m}$, around 1 to $2 \mathrm{mJy}$ for these 


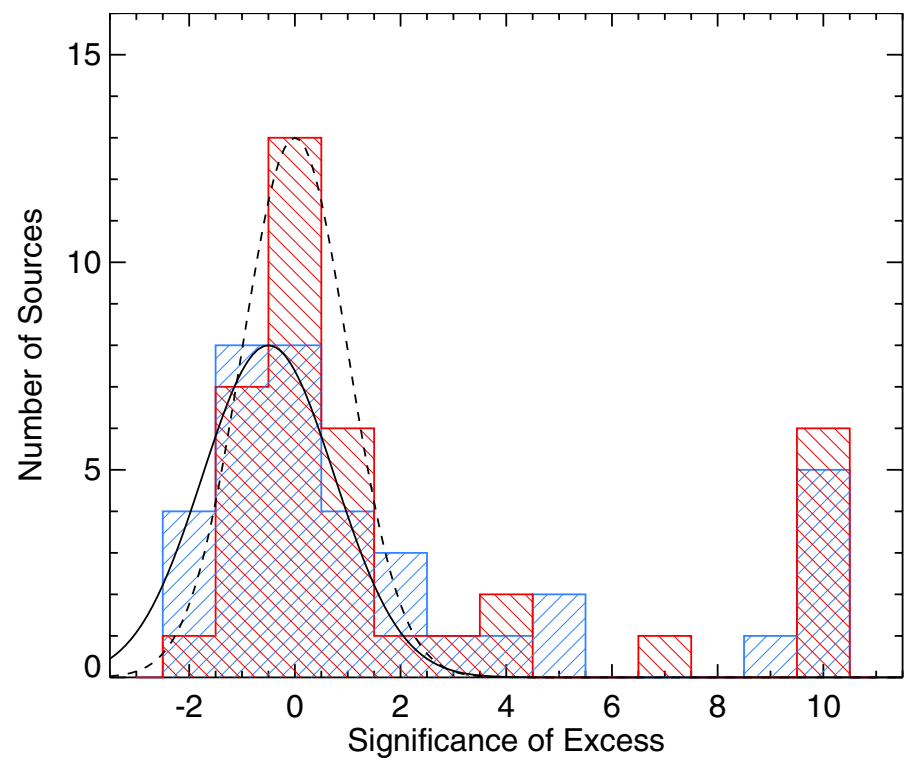

Fig. 1. Distribution of the significances presented in Table 2 at $100 \mu \mathrm{m}$ (in blue, hatching top right to bottom left) and $160 \mu \mathrm{m}$ (in red, hatching top left to bottom right). Debris disc stars with $\chi$ values higher than ten have been added to the right-hand side bin.

stars. If the magnitude of this effect were to be dependent on stellar spectral type, it would also broaden the $\chi$ distribution. The negative bias would be statistically significant should the underlying distribution be Gaussian. Because of the many unknowns in this problem, we adopted the conservative approach of noting that the sample standard deviation is larger than the negative absolute value.

Examining only the FGK stars (three of the 37 stars, one with an excess, are M dwarfs), we obtain a statistical incidence of circumstellar dust of $29.4 \pm 9.3 \%$ (10/34), consistent with the general incidence of $20.2 \pm 2.0 \%$ from the DUNES survey (Eiroa et al. 2013). Previous Spitzer results for 45 FGK exoplanet hosts record an incidence of $20.8_{-4.6}^{+7.0} \%$ (Trilling et al. 2008) and $14.5 \pm 3.5 \% 117$ FGKM stars by Kóspál et al. (2009). It should be noted that the detection frequencies quoted for various disc surveys are strongly dependent on the sensitivities, samples, and observing strategies adopted in each. Comparable incidences between surveys may therefore be coincidental due to survey differences.

From our analysis of the entire sample, we have detected discs with fractional luminosities in the range $2.4 \times 10^{-6}$ to $4.1 \times 10^{-4}$ and temperatures from $20 \mathrm{~K}$ to $80 \mathrm{~K}$. The $3 \sigma$ upper limits on the non-detections typically constrain the fractional luminosity to a $\sim$ few $\times 10^{-6}$, equivalent to $<10^{-5} M_{\oplus}$. The range of dust temperatures is broader than might be expected for typical dust temperatures for debris discs, which have the peak of their emission in the far-infrared. In the unusually cold case of HD 210277, the dust temperature can be ascribed to the nature of the source, which is a candidate "cold debris disc" (Eiroa et al. 2011; Krivov et al. 2013). Four of the circumstellar discs are new detections by Herschel: HD 20794, HD 40307, GJ 581, and HD 210277, whilst two more have been confirmed by Herschel DUNES after marginal detection by Spitzer: HD 19994 and HD 117176. Several of the Herschel discovered discs are covered in individual papers; for example HD 210277 has been identified as one of the DUNES "cold debris disc" candidates in Eiroa et al. (2011), HD 20794 in Wyatt et al. (2012) and GJ 581, an M-dwarf debris disc, in Lestrade et al. (2012). The final new detection, HD 40307, was noted in the DUNES survey paper (Eiroa et al. 2013).

\section{Discussion}

\subsection{Contamination}

A critical problem with the attribution of an infrared excess to circumstellar dust is the chance of contamination by alignment along the line of sight with a background source.

The likelihood that one (or more) of the debris discs in the sample have been spuriously identified as such was calculated from the contamination probabilities within both a radius equivalent to one beam half width at half maximum (HWHM) and the positional offset between the expected and observed source position of all the debris disc stars. The source number counts in DEBRIS survey fields from Sibthorpe et al. (2012) (their equation Eq. (2)) were used to quantify the background source number density at $100 \mu \mathrm{m}$ and $160 \mu \mathrm{m}$ for sources with flux densities equal to or brighter than the observed excesses of the debris disc stars presented here. First, the probability of confusion for each individual source, $n$, was calculated, including the specific $\mathrm{S} / \mathrm{N}$ and flux density, giving $P_{n \text {,conf }}$. Second, the probability that none of the sources were confused, $P_{\text {none }}$, was calculated by multiplication of the probabilities for the individual sources, that is $P_{\text {none }}=P_{1, \text { conf }} \times P_{2, \text { conf }} \times \ldots \times P_{n, \text { conf }}$. The probability of confusion for the whole sample is then simply $1-P_{\text {none }}$.

Within one beam HWHM, the probability of contamination of at least one source amongst the 37 is $1.4 \%$ at $100 \mu \mathrm{m}$ and $8.2 \%$ at $160 \mu \mathrm{m}$. Within the maximum position offset (6.7"), the probability of confusion increases to $4.8 \%$ at $100 \mu \mathrm{m}$ and $11.0 \%$ at $160 \mu \mathrm{m}$. In the worst case, based on the maximum offset radius, two stars at $100 \mu \mathrm{m}$ and four stars at $160 \mu \mathrm{m}$ out of the 37 could be the result of contamination.

\subsection{Correlations}

We have plotted the calculated dust fractional luminosities (or upper limits) of the exoplanet stars against several physical parameters of the host stars and exoplanets to search for trends in the sample. For Figs. 2d-f we adopted the same exoplanet parameters to characterise each system as used in Maldonado et al. (2012), so a comparison between our findings and theirs can be made more easily. We categorised the type of exoplanet system (low-mass, hot Jupiter, or cold Jupiter) according to the planet mass where $M_{\text {Planet }}>30 M_{\oplus}$ is a giant planet and a system with a planet of $R_{\text {Planet }}<0.1 \mathrm{AU}$ and $M_{\text {Planet }}>30 M_{\oplus}$ defines a hotJupiter system. Within the sample there are 11 low-mass planet systems, five hot-Jupiter systems, and 21 cold-Jupiter systems. Although no cold dust emission from HD 69830's circumstellar disc has been detected, it is included as a debris disc star in the statistics but is not marked as an excess source in the plots in Fig. 2, which were based on far-infrared emission alone. To quantify the significance of any trend observed in the figures we used the Fisher exact probability test, which has the virtue (compared with a $\chi^{2}$ test) of producing meaningful results even for small sample sizes $(N<5)$, but the test only gives a probability in support of the null hypothesis (i.e. that both samples are from the same underlying distribution). For example, comparing the number of debris disc stars around the FGK exoplanet host stars in this sample (34 stars, 10 discs) with the Spitzer and Herschel DUNES frequencies (Trilling et al. 2008; Eiroa et al. 2013), we find that the resulting $p$-values are 0.19 and 0.34 , respectively. The exoplanet host sample is not significantly different from 


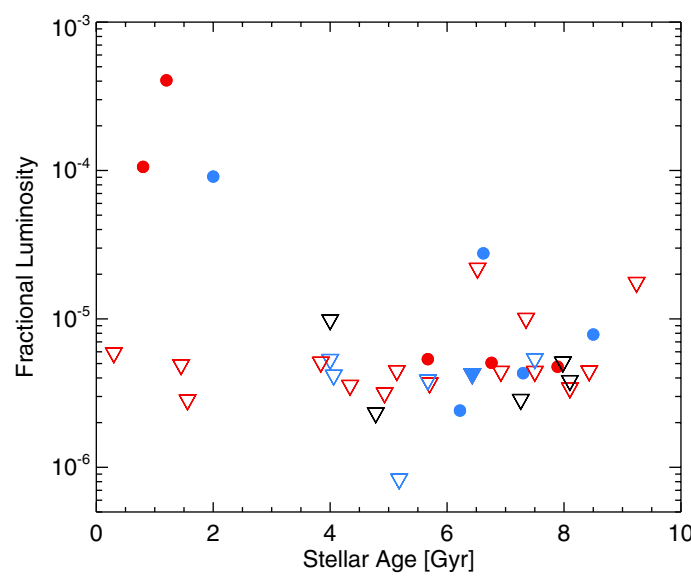

(a) Ca II age vs. fractional luminosity.

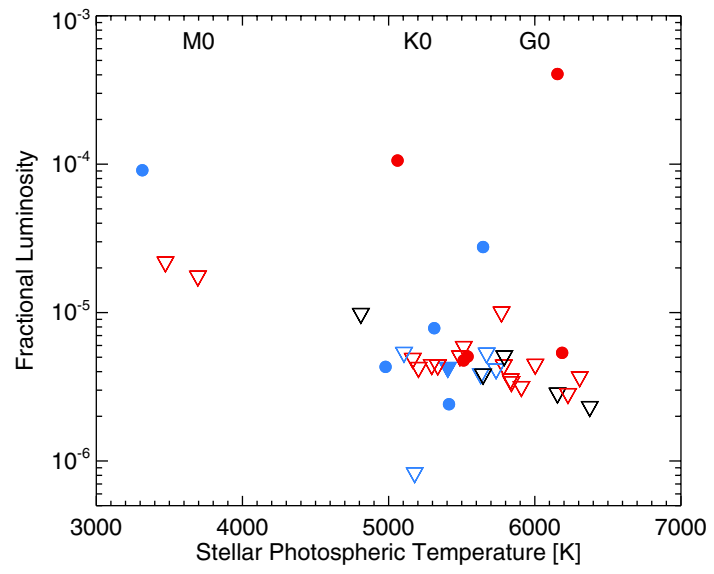

(c) Photospheric temperature vs. fractional luminosity.

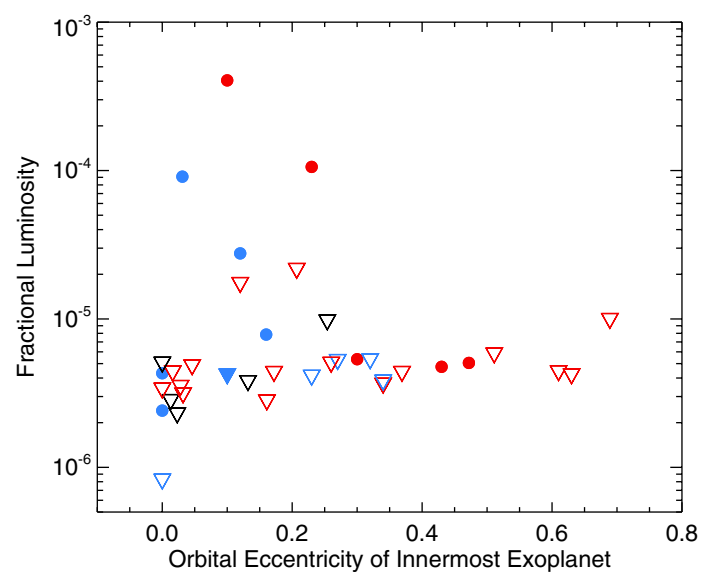

(e) Orbital eccentricity of the innermost exoplanet vs. fractional luminosity.

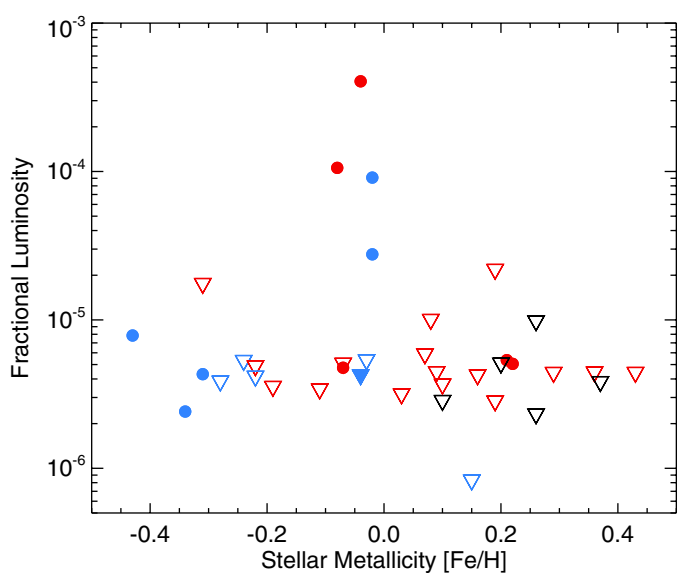

(b) Metallicity vs. fractional luminosity.

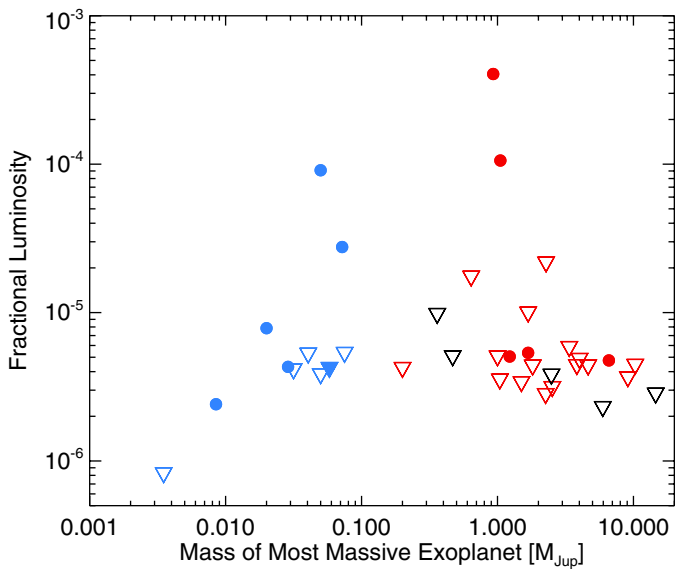

(d) Mass of most massive exoplanet vs. fractional luminosity.

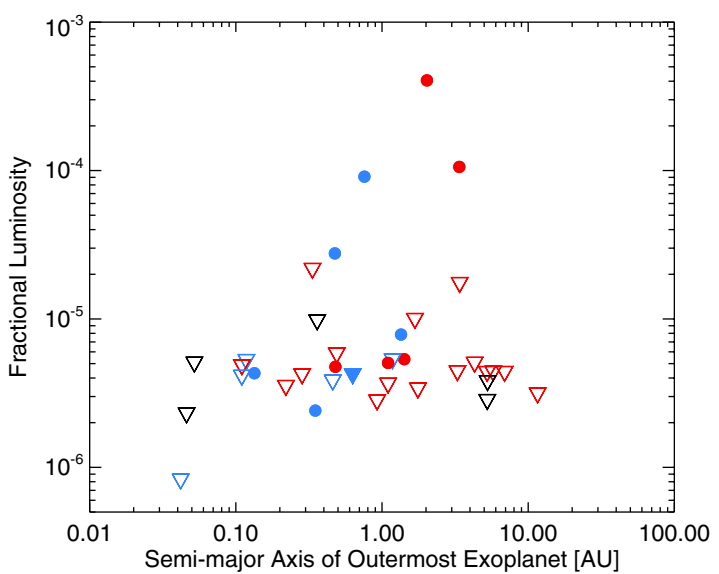

(f) Semi-major axis of the outer exoplanet orbit vs. fractional luminosity.

Fig. 2. Filled circles denote cool debris disc systems, whilst open triangles denote fits to the $3 \sigma$ upper limits at $100 \mu \mathrm{m}$. HD 69830, having a warm debris disc, is denoted as a filled blue triangle to distinguish it from the other far-infrared non-detections. Red data points are cool-giant-planet systems, black data points are hot-giant-planet systems, whilst blue data points are low-mass planet systems.

these larger samples and can be presumed to have been drawn from the same underlying population, despite the inherent biases of being composed solely of exoplanet host stars and the difference in sensitivity of the Spitzer MIPS and Herschel PACS based surveys.
The relationship between stellar age and fractional luminosity for circumstellar discs, presented in Fig. 2a, has been explored for a broad range of ages for example by Decin et al. (2003), Hernández et al. (2007), and Wyatt et al. (2007). In this work we have used stellar ages derived from $\mathrm{Ca}$ II $\mathrm{H}$ and 
K activity and X-ray luminosity taken from Eiroa et al. (2013) for the DUNES observed sources or calculated in the same manner as the DUNES sources, but based on publicly available data (Maldonado \& Sanz Forcada, priv. comm.). From binning the sample into three broad age ranges, we find that the incidence of dust weakly decreases with age, albeit with error bars large enough that the incidence could be constant across the bins considered here, from $50 \pm 29 \%(t<3.5 \mathrm{Gyr})$ to $28 \pm 12 \%$ $(t=3.5-7.0 \mathrm{Gyr})$ to $25 \pm 14 \%(t>7.0 \mathrm{Gyr})$. The decay of debris discs around Sun-like stars is examined in greater detail in Kains et al. (2011).

The sample of exoplanet host stars is almost evenly divided between sub- and supra-Solar metallicity, with 18 and 19 stars in each subsample, which is shown in Fig. 2b. Of the stars with higher metallicities than the Sun, five host a hot-Jupiter planet, whilst none of the 18 low metallicity stars have hot Jupiters, giving a $p$-value of 0.04 , that is the hot Jupiters and other planets do not come from the same underlying distribution, therefore we are able to identify the well-known correlation between increased stellar metallicity and hot Jupiters from our data set (Fischer \& Valenti 2005; Greaves et al. 2006; Maldonado et al. 2012). From comparing the distribution of hot and cold Jupiters versus metallicity, we find a $p$-value of 0.58 , which means that we cannot differentiate between the stars with wide and close orbiting Jovian exoplanets from this sample.

All the low-mass planetary systems (i.e. those where the most massive planet is $<30 M_{\oplus}$ ) except $\alpha$ Cen B have host stars with sub-Solar metallicity (10/18), consistent with Jenkins et al. (2013). Stars hosting low-mass planets have not been found to be preferentially metal rich, unlike stars hosting high-mass planets (Santos et al. 2001; Ghezzi et al. 2010; Buchhave et al. 2011; Mayor et al. 2011). By comparing the incidence of low-mass and high-mass planets around stars with sub- or supra-Solar metallicity, we obtain a probability $p$-value of 0.001 , suggesting that the low-mass and high-mass planet stars are not drawn from the same underlying population. This correlation between low-mass planets and low-metallicity stars could be the product of two phenomena: in a low-metallicity disc there will be fewer solids from which to form massive planetary cores that go on to become gas giant planets, making such planets rarer around those stars (Greaves et al. 2007; Wyatt et al. 2007); additionally, protoplanetary discs with lower metallicity are thought to be dispersed more easily and quickly because of the lower optical depth, so $\mathrm{UV}$ and X-rays penetrate farther into the disc and cause the gas loss through winds to be stronger, such that any nascent giant planet must capture its gaseous envelope more quickly because the disc dispersal occurs more rapidly in such systems, thereby limiting the number of gas giants that will form (Yasui et al. 2009; Ercolano \& Clarke 2010).

We find an incidence of debris around 10/18 stars in the lowmetallicity group and 2/19 in the high-metallicity group. This implies an anti-correlation between the presence of debris and metallicity, with a $p$-value of 0.005 . The presence of brighter debris discs around low-metallicity stars could be inferred to represent the inability of the low-mass planets to scatter the dust producing planetesimals near their formation region, or via migration from a larger initial semi-major axis, as effectively as a gas giant, as proposed in Wyatt et al. (2012). The observed anticorrelation may therefore not be directly related to the properties of the debris disc and stellar metallicity, but could be a consequence of the type of planets that form around low-metallicity stars as stated above and can be explained within the context of a model in which planets form through core accretion.
The effect of the stellar temperature on the determination of the upper limit to the dust fractional luminosity can be seen in Fig. 2c. For the coolest stars in the sample, we are limited to disc brightnesses of $\geq 2 \times 10^{-5}$, whilst at the hotter end we can put better constraints of $\geq 2 \times 10^{-6}$ on the dust present around these stars. For most stars in our sample we obtained $3 \sigma$ upper limits on the fractional luminosity of a few $\times 10^{-6}$, that is several ten times higher than that expected of the EKB (Vitense et al. 2012). There is no visible correlation between the stellar photosphere temperature and the presence of dust around the star. Dividing the sample by spectral type, we find debris discs around $33.3 \pm 23.6 \%$ of $F$ stars $(2 / 6), 27.3 \pm 11.1 \%$ of $G$ stars $(6 / 22)$, $33.3 \pm 23.6 \%$ of $\mathrm{K}$ stars $(2 / 6)$, and $33.3 \pm 33.3 \%$ of $\mathrm{M}$ stars $(1 / 3)$. These values are consistent with the measurements from surveys with much larger samples, e.g. Trilling et al. (2008), Kóspál et al. (2009) and Eiroa et al. (2013) for FGK stars, and Lestrade et al. (2006, 2009), Gautier et al. (2007) for M stars.

As seen in Fig. 2d, the exoplanets around the Herschel discovered debris disc stars all have masses $M_{\text {Planet }}<30 M_{\oplus}$. Comparing the most massive exoplanet in each system with the dust fractional luminosity reveals that the stars with brighter discs generally have a low-mass planet $\left(M_{\text {Planet }}<30 M_{\oplus}\right)$, being 6/11 stars for the low-mass subset or $5 / 26$ for the remainder of the sample. The $p$-value for the comparison of these two subsamples is 0.05 . Stars with only low-mass planets are more likely to harbour debris discs than stars with Jovian planet(s), consistent with the prediction of (Raymond et al. 2012) and the findings of Maldonado et al. (2012), thereby confirming the trend suggested in Wyatt et al. (2012) (based on a smaller sample of G stars).

The relationship between eccentricity, characterised by the orbital eccentricity of the innermost exoplanet, and fractional luminosity illustrated in Fig. 2e appears to favour the presence of debris discs around stars with low-eccentricity planetary systems $(e<0.2)$. Splitting the sample at $e=0.2$, we find a higher incidence of dust in low-eccentricity systems (7/20) over higheccentricity systems (4/17), agreeing with the predictions (for giant planets) in Raymond et al. (2011, 2012) - see Figs. 13 and 18 in Raymond et al. (2011), and similarly consistent with the results of Maldonado et al. (2012). However, putting these subsamples to the test, we find that the $p$-value is 0.49 , which is an inconclusive result, but suggests that the two groups are more similar than not, which may be interpreted as illustrating that the known exoplanets in these systems have little dynamical influence on the visible debris.

Similarly, a comparison of the fractional luminosities of systems with hot Jupiters and those with cold Jupiters in Fig. $2 f$ is suggestive that cold Jupiter systems have a stronger tendency to host debris discs. We see that none of the five subsets of hot Jupiter planet host stars are observed to have excess emission, whilst five of the 21 cold Jupiter planet host stars do have a detectable debris disc. In this case the Fischer test again returns an intermediate result, whose $p$-value is 0.54 . We therefore cannot confirm the trend identified in Maldonado et al. (2012) between cool giant planets and fainter debris discs based on the observations analysed here.

\section{Conclusions}

We have presented Herschel PACS observations of 37 nearby exoplanet systems from the DUNES and DEBRIS samples aimed at searching for exo-EKB analogues. Excess emission attributable to the presence of a circumstellar debris disc was observed around ten of these stars; for the remaining stars we found 
no evidence of significant excess emission, including the nonmeasurement of cold emission around HD 69830, providing a tighter upper limit to any possible cold dust in that system. We improved on the upper limits for dust detection for the stars in this sample by a factor of two over previous Spitzer observations, constraining the possible flux from cold dust in all observed systems to at worst two orders of magnitude higher than that of the $\mathrm{EKB}$ and in several cases at levels similar to that of the EKB.

We found incidences of $\sim 30 \%$ for cool debris discs around exoplanet host stars from the sample examined here, irrespective of the spectral type. Due to the large uncertainties in this measurement (from the small sample sizes), these values are in fact consistent with the incidence of debris discs measured by DUNES $20.2 \pm 2.0 \%$ (Eiroa et al. 2013). The incidence of debris is seen to decrease around older stars, again with large uncertainties.

We identified several trends between the stellar metallicity, the presence of a debris disc, and the mass of the most massive exoplanet around the star. We found that low-metallicity stars are more likely to host low-mass planets, low-metallicity stars are also more likely to have a detectable debris disc, and that lowmass planets are more likely to be associated with a detectable debris disc. This is consistent with what would be expected from the core-accretion planet formation model. We combined these trends and developed a picture for these systems in which the gas is stripped from the protoplanetary disc too quickly for Jovianmass planets to form and the resulting low-mass planets cannot scatter planetesimals as strongly as more massive planets if they form in situ, or as they migrate through a disc from a larger initial semi-major axis (Maldonado et al. 2012; Wyatt et al. 2012).

Furthermore, we found no significant evidence for a trend relating the eccentricity of the innermost planet with the fractional luminosity, suggesting that the known exoplanets in these systems have little influence on the visible presence of dust, which is expected because the two components are well separated. We also found no evidence to support the proposed trend between cold Jupiters and lower dust luminosities proposed in Maldonado et al. (2012), though this is expected because the sample analysed here is only a subset of those from Maldonado et al.'s work, and the newly discovered Herschel debris discs have been found exclusively around low-mass planet host stars.

As an extension to this analysis, a companion paper is in preparation, which will compare the exoplanet samples presented here with unbiased control samples of stars with exoplanets or a debris disc, or without either. This future paper will search for differences in the incidence of dusty debris between these different subsamples. In future work, observations from the Herschel open time programme "Search for Kuiper Belts Around Radial-Velocity Planet Stars" (SKARPS; Bryden et al. 2013; Kennedy et al. 2013) will be used to increase the number of radial velocity planet host stars for which far-infrared fluxes are available, which will clarify, and hopefully support, the trends seen between dust, planet, and stellar properties in this, and earlier, works.

Acknowledgements. This research has made use of NASA's Astrophysics Data System Bibliographic Services. This research has made use of the SIMBAD database, operated at CDS, Strasbourg, France. This research has made use of the Exoplanet Orbit Database and the Exoplanet Data Explorer at exoplanets.org, and the Extrasolar Planets Encyclopedia at exoplanets.eu. J.P.M., C.E., J.M. and B.M. are partially supported by Spanish grant AYA 2011-26202. This work was supported by the European Union through ERC grant number no. 279973 (GMK and $\mathrm{MCW}$ ) and has been partially supported by Spitzer grant OT1_amoromar_1 (AMM).

\section{References}

Anglada-Escudé, G., \& Tuomi, M. 2012, A\&A, 548, A58 Auvergne, M., Bodin, P., Boisnard, L., et al. 2009, A\&A, 506, 411

Avrett, E. H. 2003, in Current Theoretical Models and Future High Resolution Solar Observations: Preparing for ATST, eds. A. A. Pevtsov, \& H. Uitenbroek, ASP Conf. Ser., 286, 419

Backman, D. E., \& Paresce, F. 1993, in Protostars and Planets III, eds. E. H. Levy, \& J. I. Lunine, 1253

Backman, D. E., Dasgupta, A., \& Stencel, R. E. 1995, ApJ, 450, L35

Bailey, J., Butler, R. P., Tinney, C. G., et al. 2009, ApJ, 690, 743

Bakos, G. Á., Lázár, J., Papp, I., Sári, P., \& Green, E. M. 2002, PASP, 114, 974

Balog, Z., Müller, T., Nielbock, M., et al. 2014, Exp. Astron., in press [arXiv: 1309.6099]

Beichman, C. A., Bryden, G., Gautier, T. N., et al. 2005, ApJ, 626, 1061

Bertone, E., Buzzoni, A., Chávez, M., \& Rodríguez-Merino, L. H. 2004, AJ, 128,829

Bond, J. C., Lauretta, D. S., \& O’Brien, D. P. 2010, Icarus, 205, 321

Bonfils, X., Forveille, T., Delfosse, X., et al. 2005, A\&A, 443, L15

Booth, M., Kennedy, G., Sibthorpe, B., et al. 2013, MNRAS, 428, 1263

Borucki, W. J., Koch, D. G., Basri, G., et al. 2011, ApJ, 728, 117

Brogi, M., Snellen, I. A. G., de Kok, R. J., et al. 2012, Nature, 486, 502

Brott, I., \& Hauschildt, P. H. 2005, in The Three-Dimensional Universe with Gaia, eds. C. Turon, K. S. O'Flaherty, \& M. A. C. Perryman, ESA SP, 576, 565

Bryden, G., Beichman, C. A., Carpenter, J. M., et al. 2009, ApJ, 705, 1226

Bryden, G., Krist, J. E., Stapelfeldt, K. R., et al. 2013, in Am. Astron. Soc. Meet. Abstracts, 221, 144.24

Buchhave, L. A., Bakos, G. Á., Hartman, J. D., et al. 2011, ApJ, 733, 116

Butler, R. P., \& Marcy, G. W. 1996, ApJ, 464, L153

Butler, R. P., Marcy, G. W., Williams, E., Hauser, H., \& Shirts, P. 1997, ApJ, 474, L115

Butler, R. P., Marcy, G. W., Fischer, D. A., et al. 1999, ApJ, 526, 916

Butler, R. P., Tinney, C. G., Marcy, G. W., et al. 2001, ApJ, 555, 410

Butler, R. P., Marcy, G. W., Vogt, S. S., et al. 2003, ApJ, 582, 455

Butler, R. P., Wright, J. T., Marcy, G. W., et al. 2006, ApJ, 646, 505

Cassan, A., Kubas, D., Beaulieu, J.-P., et al. 2012, Nature, 481, 167

Chen, C. H., Sheehan, P., Watson, D. M., Manoj, P., \& Najita, J. R. 2009, ApJ, 701,1367

Cochran, W. D., Hatzes, A. P., Butler, R. P., \& Marcy, G. W. 1997, ApJ, 483, 457

Corder, S., Carpenter, J. M., Sargent, A. I., et al. 2009, ApJ, 690, L65

Cumming, A., Marcy, G. W., \& Butler, R. P. 1999, ApJ, 526, 890

Decin, G., Dominik, C., Waters, L. B. F. M., \& Waelkens, C. 2003, ApJ, 598, 636

Delfosse, X., Forveille, T., Mayor, M., et al. 1998, A\&A, 338, L67

Dumusque, X., Pepe, F., Lovis, C., et al. 2012, Nature, 491, 207

Eddy, J. A., Léna, P. J., \& MacQueen, R. M. 1969, Sol. Phys., 10, 330

Eiroa, C., Fedele, D., Maldonado, J., et al. 2010, A\&A, 518, L131

Eiroa, C., Marshall, J. P., Mora, A., et al. 2011, A\&A, 536, L4

Eiroa, C., Marshall, J. P., Mora, A., et al. 2013, A\&A, 555, A11

Endl, M., Robertson, P., Cochran, W. D., et al. 2012, ApJ, 759, 19

Ercolano, B., \& Clarke, C. J. 2010, MNRAS, 402, 2735

Faherty, J. K., Burgasser, A. J., West, A. A., et al. 2010, AJ, 139, 176

Fischer, D. A., \& Valenti, J. 2005, ApJ, 622, 1102

Fischer, D. A., Marcy, G. W., Butler, R. P., Vogt, S. S., \& Apps, K. 1999, PASP, 111,50

Fischer, D. A., Marcy, G. W., Butler, R. P., Laughlin, G., \& Vogt, S. S. 2002, ApJ, 564, 1028

Fischer, D. A., Butler, R. P., Marcy, G. W., Vogt, S. S., \& Henry, G. W. 2003, ApJ, 590, 1081

Fischer, D. A., Marcy, G. W., Butler, R. P., et al. 2008, ApJ, 675, 790

Fischer, D. A., Marcy, G. W., \& Spronck, J. F. P. 2014, ApJS, 210, 5

Forveille, T., Bonfils, X., Delfosse, X., et al. 2011, A\&A, submitted [arXiv: 1109.2505]

Galland, F., Lagrange, A.-M., Udry, S., et al. 2005, A\&A, 444, L21

Gautier, III, T. N., Rieke, G. H., Stansberry, J., et al. 2007, ApJ, 667, 527

Ghezzi, L., Cunha, K., Smith, V. V., et al. 2010, ApJ, 720, 1290

Giacobbe, P., Damasso, M., Sozzetti, A., et al. 2012, MNRAS, 424, 3101

Gomes, R., Levison, H. F., Tsiganis, K., \& Morbidelli, A. 2005, Nature, 435, 466 Goździewski, K., Maciejewski, A. J., \& Migaszewski, C. 2007, ApJ, 657, 546

Greaves, J. S., \& Wyatt, M. C. 2010, MNRAS, 404, 1944

Greaves, J. S., Fischer, D. A., \& Wyatt, M. C. 2006, MNRAS, 366, 283

Greaves, J. S., Fischer, D. A., Wyatt, M. C., Beichman, C. A., \& Bryden, G. 2007, MNRAS, 378, L1

Gregory, P. C., \& Fischer, D. A. 2010, MNRAS, 403, 731

Griffin, M. J., Abergel, A., Abreu, A., et al. 2010, A\&A, 518, L3 
Gurnett, D. A., Ansher, J. A., Kurth, W. S., \& Granroth, L. J. 1997, Geophys. Res. Lett., 24, 3125

Han, D., Poppe, A. R., Piquette, M., Grün, E., \& Horányi, M. 2011, Geophys. Res. Lett., 38, 24102

Hatzes, A. P. 2013, ApJ, 770, 133

Hatzes, A. P., Cochran, W. D., McArthur, B., et al. 2000, ApJ, 544, L145

Hernández, J., Hartmann, L., Megeath, T., et al. 2007, ApJ, 662, 1067

Holland, W. S., Greaves, J. S., Zuckerman, B., et al. 1998, Nature, 392, 788

Horner, J., \& Jones, B. W. 2009, Int. J. Astrobiol., 8, 75

Horner, J., \& Jones, B. W. 2010, Int. J. Astrobiol., 9, 273

Horner, J., Mousis, O., Petit, J.-M., \& Jones, B. W. 2009, Planet. Space Sci., 57, 1338

Howard, A. W., Johnson, J. A., Marcy, G. W., et al. 2011, ApJ, 730, 10

Hughes, A. M., Wilner, D. J., Andrews, S. M., et al. 2011, ApJ, 740, 38

Hughes, A. M., Wilner, D. J., Mason, B., et al. 2012, ApJ, 750, 82

Izidoro, A., de Souza Torres, K., Winter, O. C., \& Haghighipour, N. 2013, ApJ, 767, 54

Janson, M., Brandt, T. D., Moro-Martín, A., et al. 2013, ApJ, 773, 73

Jenkins, J. S., Jones, H. R. A., Tuomi, M., et al. 2013, ApJ, 766, 67

Jones, H. R. A., Paul Butler, R., Tinney, C. G., et al. 2002, MNRAS, 333, 871

Kains, N., Wyatt, M. C., \& Greaves, J. S. 2011, MNRAS, 414, 2486

Kalas, P., Graham, J. R., Chiang, E., et al. 2008, Science, 322, 1345

Kennedy, G. M., Wyatt, M. C., Bryden, G., Wittenmyer, R., \& Sibthorpe, B. 2013, MNRAS

Kóspál, Á., Ardila, D. R., Moór, A., \& Ábrahám, P. 2009, ApJ, 700, L73

Krivov, A. V. 2010, Res. Astron. Astrophys., 10, 383

Krivov, A. V., Eiroa, C., Löhne, T., et al. 2013, ApJ, 772, 32

Kürster, M., Endl, M., Els, S., et al. 2000, A\&A, 353, L33

Kuzuhara, M., Tamura, M., Kudo, T., et al. 2013, ApJ, 774, 11

Lagrange, A.-M., Bonnefoy, M., Chauvin, G., et al. 2010, Science, 329, 57

Lestrade, J.-F., Wyatt, M. C., Bertoldi, F., Dent, W. R. F., \& Menten, K. M. 2006, A\&A, 460, 733

Lestrade, J.-F., Wyatt, M. C., Bertoldi, F., Menten, K. M., \& Labaigt, G. 2009, A\&A, 506, 1455

Lestrade, J.-F., Matthews, B. C., Sibthorpe, B., et al. 2012, A\&A, 548, A86

Liseau, R., Montesinos, B., Olofsson, G., et al. 2013, A\&A, 549, L7

Löhne, T., Augereau, J.-C., Ertel, S., et al. 2012, A\&A, 537, A110

Lovis, C., Mayor, M., Pepe, F., et al. 2006, Nature, 441, 305

Lyra, W., \& Kuchner, M. 2013, Nature, 499, 184

Maldonado, J., Eiroa, C., Villaver, E., Montesinos, B., \& Mora, A. 2012, A\&A, 541, A40

Mamajek, E. E., \& Hillenbrand, L. A. 2008, ApJ, 687, 1264

Marcy, G. W., \& Butler, R. P. 1996, ApJ, 464, L147

Marcy, G. W., \& Butler, R. P. 2000, PASP, 112, 137

Marcy, G. W., Butler, R. P., Vogt, S. S., Fischer, D., \& Lissauer, J. J. 1998, ApJ, 505, L147

Marcy, G. W., Butler, R. P., Vogt, S. S., Fischer, D., \& Liu, M. C. 1999, ApJ, 520,239

Marcy, G. W., Butler, R. P., Fischer, D., et al. 2001, ApJ, 556, 296

Marcy, G. W., Butler, R. P., Fischer, D. A., et al. 2002, ApJ, 581, 1375

Marcy, G. W., Butler, R. P., Vogt, S. S., et al. 2005, ApJ, 619, 570

Marois, C., Macintosh, B., Barman, T., et al. 2008, Science, 322, 1348

Marshall, J. P., Löhne, T., Montesinos, B., et al. 2011, A\&A, 529, A117

Matthews, B. C., Sibthorpe, B., Kennedy, G., et al. 2010, A\&A, 518, L135

Mayor, M., \& Queloz, D. 1995, Nature, 378, 355

Mayor, M., \& Queloz, D. 2012, New Astron. Rev., 56, 19

Mayor, M., Udry, S., Naef, D., et al. 2004, A\&A, 415, 391

Mayor, M., Udry, S., Lovis, C., et al. 2009, A\&A, 493, 639

Mayor, M., Marmier, M., Lovis, C., et al. 2011, A\&A, submitted [arXiv: 1109.2497]

McArthur, B. E., Endl, M., Cochran, W. D., et al. 2004, ApJ, 614, L81

McCarthy, C., Butler, R. P., Tinney, C. G., et al. 2004, ApJ, 617, 575

Meschiari, S., Laughlin, G., Vogt, S. S., et al. 2011, ApJ, 727, 117

Morales, F. Y., Rieke, G. H., Werner, M. W., et al. 2011, ApJ, 730, L29

Moran, S. M., Kuchner, M. J., \& Holman, M. J. 2004, ApJ, 612, 1163

Morbidelli, A., Chambers, J., Lunine, J. I., et al. 2000, Meteoritics and Planetary, Science, 35, 1309

Moro-Martin, A. 2013, Dusty Planetary Systems, eds. T. D. Oswalt, L. M. French, \& P. Kalas, 431

Moro-Martín, A., Carpenter, J. M., Meyer, M. R., et al. 2007, ApJ, 658, 1312

Muterspaugh, M. W., Lane, B. F., Kulkarni, S. R., et al. 2010, AJ, 140, 1657

Naef, D., Mayor, M., Pepe, F., et al. 2001, A\&A, 375, 205

Naef, D., Mayor, M., Lo Curto, G., et al. 2010, A\&A, 523, A15

Noyes, R. W., Jha, S., Korzennik, S. G., et al. 1997, ApJ, 483, L111

O'Brien, D. P., Morbidelli, A., \& Levison, H. F. 2006, Icarus, 184, 39

Ott, S. 2010, in Astronomical Data Analysis Software and Systems XIX, eds.

Y. Mizumoto, K.-I. Morita, \& M. Ohishi, ASP Conf. Ser., 434, 139

Pepe, F., Correia, A. C. M., Mayor, M., et al. 2007, A\&A, 462, 769
Pepe, F., Lovis, C., Ségransan, D., et al. 2011, A\&A, 534, A58 Perryman, M. 2011, The Exoplanet Handbook

Piétu, V., di Folco, E., Guilloteau, S., Gueth, F., \& Cox, P. 2011, A\&A, 531, L2

Pilbratt, G. L., Riedinger, J. R., Passvogel, T., et al. 2010, A\&A, 518, L1 Poglitsch, A., Waelkens, C., Geis, N., et al. 2010, A\&A, 518, L2

Pollacco, D. L., Skillen, I., Collier Cameron, A., et al. 2006, PASP, 118, 1407

Poppe, A., James, D., Jacobsmeyer, B., \& Horányi, M. 2010, Geophys. Res. Lett., 37, 11101

Queloz, D., Mayor, M., Weber, L., et al. 2000, A\&A, 354, 99

Rameau, J., Chauvin, G., Lagrange, A.-M., et al. 2013, ApJ, 772, L15

Raymond, S. N., Quinn, T., \& Lunine, J. I. 2004, Icarus, 168, 1

Raymond, S. N., Quinn, T., \& Lunine, J. I. 2006, Icarus, 183, 265

Raymond, S. N., Quinn, T., \& Lunine, J. I. 2007, Astrobiology, 7, 66

Raymond, S. N., Armitage, P. J., Moro-Martín, A., et al. 2011, A\&A, 530, A62

Raymond, S. N., Armitage, P. J., Moro-Martín, A., et al. 2012, A\&A, 541, A11

Reiners, A., Bean, J. L., Huber, K. F., et al. 2010, ApJ, 710, 432

Rivera, E. J., Lissauer, J. J., Butler, R. P., et al. 2005, ApJ, 634, 625

Rivera, E. J., Laughlin, G., Butler, R. P., et al. 2010, ApJ, 719, 890

Rodler, F., Del Burgo, C., Witte, S., et al. 2011, A\&A, 532, A31

Santos, N. C., Israelian, G., \& Mayor, M. 2001, A\&A, 373, 1019

Santos, N. C., Bouchy, F., Mayor, M., et al. 2004, A\&A, 426, L19

Sanz-Forcada, J., Micela, G., Ribas, I., et al. 2011, A\&A, 532, A6

Sibthorpe, B., Ivison, R. J., Massey, R. J., et al. 2012, MNRAS, L3

Swinyard, B. M., Ade, P., Baluteau, J.-P., et al. 2010, A\&A, 518, L4

Tinney, C. G., Butler, R. P., Marcy, G. W., et al. 2001, ApJ, 551, 507

Tinney, C. G., Butler, R. P., Jones, H. R. A., et al. 2011, ApJ, 727, 103

Trilling, D. E., Bryden, G., Beichman, C. A., et al. 2008, ApJ, 674, 1086

Tuomi, M., Jones, H. R. A., Jenkins, J. S., et al. 2013, A\&A, 551, A79

Udry, S., Mayor, M., Benz, W., et al. 2006, A\&A, 447, 361

Udry, S., Bonfils, X., Delfosse, X., et al. 2007, A\&A, 469, L43

van Leeuwen, F. 2007, A\&A, 474, 653

Vitense, C., Krivov, A. V., Kobayashi, H., \& Löhne, T. 2012, A\&A, 540, A30

Vogt, S. S., Butler, R. P., Marcy, G. W., et al. 2005, ApJ, 632, 638

Vogt, S. S., Wittenmyer, R. A., Butler, R. P., et al. 2010, ApJ, 708, 1366

Vogt, S. S., Butler, R. P., \& Haghighipour, N. 2012, Astron. Nachr., 333, 561

Wahhaj, Z., Liu, M. C., Nielsen, E. L., et al. 2013, ApJ, 773, 179

Walsh, K. J., Morbidelli, A., Raymond, S. N., O’Brien, D. P., \& Mandell, A. M. 2011, Nature, 475, 206

Wittenmyer, R. A., Endl, M., \& Cochran, W. D. 2007, ApJ, 654, 625

Wittenmyer, R. A., Endl, M., Cochran, W. D., Levison, H. F., \& Henry, G. W. 2009, ApJS, 182, 97

Wittenmyer, R. A., Tinney, C. G., O’Toole, S. J., et al. 2011, ApJ, 727, 102

Wright, J. T., Marcy, G. W., Fischer, D. A., et al. 2007, ApJ, 657, 533

Wright, J. T., Marcy, G. W., Butler, R. P., et al. 2008, ApJ, 683, L63

Wright, J. T., Upadhyay, S., Marcy, G. W., et al. 2009, ApJ, 693, 1084

Wyatt, M. C. 2003, ApJ, 598, 1321

Wyatt, M. C. 2008, ARA\&A, 46, 339

Wyatt, M. C., Clarke, C. J., \& Greaves, J. S. 2007, MNRAS, 380, 1737

Wyatt, M. C., Kennedy, G., Sibthorpe, B., et al. 2012, MNRAS, 424, 1206

Yasui, C., Kobayashi, N., Tokunaga, A. T., Saito, M., \& Tokoku, C. 2009, ApJ, 705,54

Zechmeister, M., Kürster, M., Endl, M., et al. 2013, A\&A, 552, A78

1 Depto. de Física Teórica, Universidad Autónoma de Madrid, Cantoblanco, 28049 Madrid, Spain

e-mail: jonathan.marshall@uam.es

2 School of Physics, University of New South Wales, Sydney, NSW 2052, Australia

3 Department of Astrophysics, Center for Astrobiology, Ctra. de Ajalvir, km 4, Torrejon de Ardoz, 28850 Madrid, Spain

4 Space Telescope Science Institute, 3700 San Martin Dr, Baltimore, MD 21218, USA

5 Institute of Astronomy (IoA), University of Cambridge, Madingley Road, Cambridge, CB3 OHA, UK

6 ESA-ESAC Gaia SOC. PO Box 78, 28691 Villanueva de la Cañada, Madrid, Spain

7 SRON Netherlands Institute for Space Research, 9747 AD Groningen, The Netherlands

8 Observatoire de Paris, CNRS, 61 Av. de l'Observatoire, 75014 Paris, France

9 INAF Observatorio Astronomico di Palermo, Piazza Parlamento 1, 90134 Palermo, Italy 
10 Department of Astrophysics, Centre for Astrobiology (CAB, CSICINTA), ESAC Campus, PO Box 78, 28691 Villanueva de la Cañada, Madrid, Spain

11 Herzberg Astronomy \& Astrophysics, National Research Council of Canada, 5071 West Saanich Rd, Victoria, BC V9E 2E7, Canada

12 University of Victoria, Finnerty Road, Victoria, BC, V8W 3P6, Canada

13 Australian Centre for Astrobiology, University of New South Wales, Sydney, NSW 2052, Australia

14 Computational Engineering and Science Research Centre, University of Southern Queensland, Toowoomba, 4350 Queensland, Australia

15 Jet Propulsion Laboratory, California Institute of Technology, Pasadena, CA 91109, USA

${ }^{16}$ Instituto Nacional de Astrofísica, Óptica y Electrónica, Luis Enrique Erro 1, Sta. Ma. Tonantzintla, Puebla, Mexico
17 SUPA, School of Physics and Astronomy, University of St. Andrews, North Haugh, St. Andrews KY16 9SS, UK

18 UK Astronomy Technology Centre, Royal Observatory Edinburgh, Blackford Hill, Edinburgh EH9 3HJ, UK

19 Institute for Astronomy, University of Edinburgh, Royal Observatory, Blackford Hill, Edinburgh EH9 3HJ, UK

20 Department of Astronomy, Stockholm University, AlbaNova University Center, Roslagstullsbacken 21, 10691 Stockholm, Sweden

21 ESA Astrophysics \& Fundamental Physics Missions Division, ESTEC/SRE-SA, Keplerlaan 1, 2201 AZ Noordwijk, The Netherlands

22 Department of Physical sciences, The Open University, Walton Hall, Milton Keynes MK7 6AA, UK

${ }^{23}$ Rutherford Appleton Laboratory, Chilton OX11 0QX, UK 
Table 2. Spitzer MIPS70 and Herschel PACS photometry along with photospheric estimates for the exoplanet host stars.

\begin{tabular}{|c|c|c|c|c|c|c|c|c|c|c|c|c|}
\hline Name & $\begin{array}{c}F_{\mathrm{MIPS}}[70] \\
{[\mathrm{mJy}]}\end{array}$ & $\begin{array}{c}F_{\mathrm{PACS}}[70] \\
{[\mathrm{mJy}]}\end{array}$ & $\begin{array}{c}F_{\star}[70] \\
{[\mathrm{mJy}]}\end{array}$ & $\begin{array}{c}F_{\mathrm{PACS}}[100] \\
{[\mathrm{mJy}]}\end{array}$ & $\begin{array}{c}F_{\star}[100] \\
{[\mathrm{mJy}]}\end{array}$ & $\begin{array}{c}F_{\mathrm{PACS}}[160] \\
{[\mathrm{mJy}]}\end{array}$ & $\begin{array}{c}F_{\star}[160] \\
{[\mathrm{mJy}]}\end{array}$ & $\chi_{100}$ & $\chi_{160}$ & $\begin{array}{c}L_{\mathrm{IR}} / L_{\star} \\
{\left[\times 10^{-6}\right]}\end{array}$ & $\begin{array}{l}R_{\text {disc }} \\
{[\mathrm{AU}]}\end{array}$ & $\begin{array}{l}T_{\text {disc }} \\
{[\mathrm{K}]}\end{array}$ \\
\hline HD 1237 & $11.2 \pm 2.5$ & & 9.56 & $4.57 \pm 1.19$ & 4.69 & $-0.72 \pm 3.36$ & 1.81 & -0.1 & -0.8 & $<6.4$ & $(45.3)$ & \\
\hline HD 3651 & $14.9 \pm 5.7$ & $21.93 \pm 1.59$ & 22.90 & $8.21 \pm 1.21$ & 11.22 & $3.76 \pm 3.43$ & 4.38 & -2.5 & -0.2 & $<4.6$ & (41.1) & \\
\hline HD 4308 & $5.2 \pm 4.4$ & $7.97 \pm 0.93$ & 8.72 & $5.23 \pm 1.00$ & 4.27 & $1.63 \pm 1.63$ & 1.67 & 1.0 & -0.0 & $<5.8$ & $(56.8)$ & \\
\hline$v$ And & $55.7 \pm 6.3$ & $\ldots$ & 60.22 & $32.78 \pm 1.67$ & 29.51 & $16.49 \pm 2.64$ & 11.53 & 2.0 & 1.9 & $<3.1$ & (103.5) & \\
\hline$q^{1}$ Eri & $863.4 \pm 58.7$ & $896.20 \pm 26.90$ & 17.48 & $897.10 \pm 26.90$ & 8.56 & $635.90 \pm 31.80$ & 3.35 & 33.0 & 19.9 & 405.0 & 25.7 & 60.9 \\
\hline$\tau$ Ceti $^{a}$ & $\ldots$ & $301.0 \pm 15.0$ & 183.50 & & 89.92 & $103.0 \pm 5.0$ & 32.20 & 4.8 & 14.2 & 7.8 & 12.2 & 67.5 \\
\hline GJ 86 & $6.9 \pm 6.8$ & $14.28 \pm 1.66$ & 18.86 & $7.07 \pm 1.30$ & 9.24 & $3.78 \pm 2.49$ & 3.61 & -1.7 & 0.1 & $<5.3$ & $(36.0)$ & \\
\hline$\iota$ Hor & $20.1 \pm 4.1$ & $\ldots$ & 18.82 & $7.34 \pm 1.25$ & 9.22 & $4.29 \pm 3.10$ & 3.55 & -1.5 & 0.2 & $<3.1$ & (69.6) & \\
\hline HD 19994 & $42.5 \pm 3.5$ & $\ldots$ & 26.43 & $39.45 \pm 1.75$ & 12.95 & $31.75 \pm 1.94$ & 5.06 & 15.1 & 13.8 & 5.4 & 91.1 & 40.8 \\
\hline HD $20794^{b}$ & $94.0 \pm 13.6$ & $97.0 \pm 5.5$ & 83.62 & $56.01 \pm 2.86$ & 40.97 & $23.37 \pm 2.42$ & 15.79 & 5.3 & 3.1 & 2.4 & 10.8 & 76.5 \\
\hline$\epsilon \mathbf{E r r i}^{a}$ & $1688.0 \pm 10.0$ & $1599.5 \pm 80.0$ & 187.60 & & 91.90 & $1120.8 \pm 56.0$ & 35.90 & 9.5 & 19.4 & 107.6 & 13.4 & 57.9 \\
\hline HD 33564 & $32.6 \pm 5.5$ & $\ldots$ & 22.04 & $12.02 \pm 2.24$ & 10.80 & $7.30 \pm 2.70$ & 4.16 & 0.5 & 1.2 & $<4.0$ & (101.4) & \\
\hline HD 39091 & $23.4 \pm 2.9$ & $\ldots$ & 16.15 & $12.26 \pm 1.84$ & 7.91 & $5.59 \pm 2.87$ & 3.05 & 2.4 & 0.9 & $<4.8$ & (69.9) & \\
\hline HD $40307^{b}$ & $17.2 \pm 4.9$ & $14.60 \pm 1.43$ & 10.01 & $8.05 \pm 0.95$ & 4.90 & $8.02 \pm 1.50$ & 1.92 & 3.3 & 4.1 & 4.3 & 24.0 & 39.9 \\
\hline HD $69830^{c}$ & $15.1 \pm 2.4$ & $\ldots$ & 19.84 & $9.33 \pm 0.72$ & 9.72 & $2.01 \pm 2.01$ & 3.80 & -0.5 & -0.9 & $<4.6$ & $(43.5)$ & \\
\hline $55 \mathrm{Cnc}$ & $19.8 \pm 4.4$ & $\ldots$ & 19.66 & $8.59 \pm 0.87$ & 9.64 & $4.29 \pm 1.92$ & 3.76 & -1.2 & 0.3 & $<4.8$ & $(43.8)$ & \\
\hline $47 \mathrm{UMa}$ & $31.4 \pm 4.2$ & $\ldots$ & 30.26 & $13.52 \pm 1.16$ & 14.83 & $2.82 \pm 2.82$ & 5.79 & -1.1 & -1.1 & $<3.4$ & (71.4) & \\
\hline HD 99492 & $7.5 \pm 6.3$ & $\ldots$ & 6.79 & $5.68 \pm 1.03$ & 3.33 & $4.57 \pm 3.96$ & 1.30 & 2.3 & 0.8 & $<10.6$ & $(32.3)$ & \\
\hline HD 102365 & $34.0 \pm 8.5$ & $\ldots$ & 41.43 & $20.89 \pm 1.43$ & 20.30 & $7.53 \pm 2.99$ & 7.83 & 0.4 & -0.1 & $<4.2$ & (51.7) & \\
\hline $61 \mathrm{Vir}$ & $185.6 \pm 16.6$ & $198.00 \pm 10.34$ & 49.77 & $156.64 \pm 8.63$ & 24.39 & $131.30 \pm 7.13$ & 9.40 & 15.3 & 17.1 & 27.6 & 22.4 & 56.1 \\
\hline 70 Vir & $79.0 \pm 8.1$ & $\ldots$ & 42.07 & $40.73 \pm 2.14$ & 20.61 & $26.97 \pm 1.39$ & 8.05 & 9.4 & 13.6 & 4.8 & 49.3 & 52.1 \\
\hline$\tau$ Boo & $33.2 \pm 6.8$ & & 38.07 & $16.97 \pm 1.37$ & 18.65 & $6.24 \pm 2.50$ & 7.29 & -1.2 & -0.4 & $<2.7$ & $(98.8)$ & \\
\hline$\alpha$ Cen B & $1012.0 \pm 667.8$ & $1490.0 \pm 280.0$ & 1510.00 & $670.0 \pm 37.0$ & 740.00 & $210.0 \pm 60.0$ & 289.00 & -1.65 & -1.26 & $<0.9$ & (37.6) & \\
\hline GJ 581 & $15.9 \pm 4.7$ & $18.90 \pm 1.40$ & 7.06 & $10.96 \pm 1.08$ & 3.46 & $18.04 \pm 3.50$ & 1.33 & 12.0 & 4.2 & 91.0 & 5.0 & 41.0 \\
\hline$\rho \mathrm{CrB}$ & $29.6 \pm 5.4$ & $\ldots$ & 23.06 & $10.59 \pm 1.14$ & 11.30 & $1.83 \pm 1.83$ & 4.41 & -0.6 & -1.4 & $<3.8$ & $(74.5)$ & \\
\hline $14 \mathrm{Her}$ & $10.6 \pm 2.5$ & $\ldots$ & 10.76 & $3.91 \pm 0.77$ & 5.27 & $1.61 \pm 1.61$ & 2.06 & -1.8 & -0.3 & $<4.8$ & (45.6) & \\
\hline HD 154345 & $11.0 \pm 9.0$ & $6.78 \pm 1.32$ & 8.16 & $3.94 \pm 0.66$ & 4.00 & $1.93 \pm 1.93$ & 1.56 & -0.1 & 0.2 & $<5.5$ & $(44.3)$ & \\
\hline$\mu$ Ara & $31.0 \pm 7.8$ & $\ldots$ & 29.08 & $15.46 \pm 2.39$ & 14.25 & $7.06 \pm 1.72$ & 5.57 & 0.5 & 0.9 & $<4.8$ & $(76.2)$ & \\
\hline HD 176051 & $31.9 \pm 5.5$ & $\ldots$ & 27.78 & $12.30 \pm 1.32$ & 13.61 & $-0.84 \pm 3.45$ & 5.25 & -1.0 & -1.8 & $<3.7$ & $(71.5)$ & \\
\hline 16 Cyg B & $30.7 \pm 7.8$ & $\ldots$ & 11.25 & $11.10 \pm 4.40$ & 5.51 & $0.00 \pm 3.06$ & 2.12 & 1.3 & -0.7 & $<10.9$ & $(63.6)$ & \\
\hline HD 189567 & $19.0 \pm 3.4$ & $10.44 \pm 2.20$ & 12.88 & $5.71 \pm 1.00$ & 6.31 & $1.85 \pm 1.85$ & 2.46 & -0.6 & -0.3 & $<4.5$ & $(57.2)$ & \\
\hline HD 192310 & $19.1 \pm 5.6$ & $\ldots$ & 29.81 & $15.52 \pm 1.26$ & 14.61 & $7.34 \pm 3.30$ & 5.63 & 0.7 & 0.5 & $<5.8$ & $(35.8)$ & \\
\hline GJ 832 & $19.5 \pm 4.1$ & $\ldots$ & 19.66 & $10.93 \pm 1.82$ & 9.63 & $0.00 \pm 3.59$ & 3.72 & 0.7 & -1.0 & $<19.0$ & (9.9) & \\
\hline HD $210277^{b}$ & $7.9 \pm 2.2$ & $\ldots$ & 9.51 & $8.50 \pm 1.00$ & 4.66 & $12.40 \pm 1.60$ & 1.82 & 3.8 & 6.6 & 5.1 & 154.9 & 22.3 \\
\hline GJ 876 & $9.5 \pm 3.1$ & $\ldots$ & 14.58 & $7.72 \pm 1.48$ & 7.14 & $4.30 \pm 3.14$ & 2.75 & 0.4 & 0.5 & $<23.7$ & $(6.4)$ & \\
\hline $51 \mathrm{Peg}$ & $28.1 \pm 4.9$ & $\ldots$ & 21.88 & $11.49 \pm 2.65$ & 10.72 & $5.00 \pm 5.00$ & 4.19 & 0.3 & 0.2 & $<5.5$ & $(66.0)$ & \\
\hline HD 217107 & $10.9 \pm 5.3$ & $\ldots$ & 12.37 & $4.69 \pm 0.90$ & 6.10 & $1.69 \pm 3.20$ & 2.30 & -1.6 & -0.2 & $<4.1$ & (60.9) & \\
\hline
\end{tabular}

Notes. Spitzer photometry from Eiroa et al. (2013) or provided by Geoff Bryden (priv. comm.). Debris disc stars are highlighted in bold; ${ }^{(a)} \chi_{100}$ estimated from model fit to 70 and $160 \mu$ m fluxes; 
Table 3. Stellar and planetary parameters of the exoplanet host star sample.

\begin{tabular}{|c|c|c|c|c|c|c|c|c|c|c|c|c|}
\hline Name & $\begin{array}{c}\text { Spectral } \\
\text { Type } \\
\end{array}$ & $\begin{array}{c}\text { Distance } \\
{[\mathrm{pc}]}\end{array}$ & $\begin{array}{c}L_{\star} \\
{\left[L_{\odot}\right]}\end{array}$ & $\begin{array}{c}T_{\star} \\
{[\mathrm{K}]} \\
\end{array}$ & {$[\mathrm{Fe} / \mathrm{H}]$} & $\begin{array}{c}\text { Ca II Age } \\
{[\mathrm{Gyr}]}\end{array}$ & $\begin{array}{c}\text { X-ray Age } \\
{[\mathrm{Gyr}]}\end{array}$ & $N_{\text {Planet }^{a}}^{a}$ & $\begin{array}{c}M \sin i^{b} \\
{\left[M_{\text {Jup }}\right]} \\
\end{array}$ & $\begin{array}{c}a^{c} \\
{[\mathrm{AU}]}\end{array}$ & $e_{\text {Planet }}{ }^{d}$ & Ref. \\
\hline HD 1237 & G8 V & $17.49 \pm 0.09$ & 0.643 & 5514 & 0.07 & 0.3 & 0.4 & 1 & $3.37 \pm 0.09$ & $0.4947 \pm 0.0083$ & $0.511 \pm 0.017$ & 1 \\
\hline HD 3651 & K0 V & $11.06 \pm 0.04$ & 0.529 & 5204 & 0.16 & 6.4 & 4.5 & 1 & $0.229 \pm 0.015$ & $0.2947 \pm 0.0049$ & $0.63 \pm 0.04$ & 2 \\
\hline HD 4308 & G3 V & $22.06 \pm 0.16$ & 1.012 & 5670 & -0.24 & 4.0 & 4.5 & 1 & $0.0405 \pm 0.005$ & $0.118 \pm 0.0009$ & $0.27 \pm 0.12$ & 3 \\
\hline$v$ And & F8 V & $13.49 \pm 0.03$ & 3.363 & 6155 & 0.10 & 7.3 & 2.9 & 3 & $4.12 \pm 0.165$ & $2.525 \pm 0.042$ & $0.013 \pm 0.016$ & 4 \\
\hline$q^{1}$ Eri & F8 V & $17.43 \pm 0.08$ & 1.523 & 6155 & -0.04 & 1.2 & 0.8 & 1 & $0.93 \pm 0.18$ & $2.03 \pm 0.15$ & $0.1 \pm 0.01$ & 5 \\
\hline$\tau$ Ceti & G8 V & $3.65 \pm 0.01$ & 0.526 & 5312 & -0.43 & 8.5 & & 5 & $0.02 \pm 0.01$ & $1.35 \pm 0.09$ & $0.16_{-0.16}^{+0.22}$ & 6 \\
\hline GJ 86 & K0 V & $10.78 \pm 0.04$ & 0.406 & 5165 & -0.22 & 1.5 & 3.6 & 1 & $4.00 \pm 0.137$ & $0.1142 \pm 0.0019$ & $0.0416 \pm 0.0072$ & 7 \\
\hline$\iota$ Hor & F8 V & $17.17 \pm 0.06$ & 1.520 & 6227 & 0.19 & 1.6 & 0.5 & 1 & $2.05 \pm 0.20$ & $0.924 \pm 0.016$ & $0.14 \pm 0.13$ & 8 \\
\hline HD 19994 & F8 V & $22.58 \pm 0.14$ & 3.848 & 6187 & 0.21 & 5.67 & 1.2 & 1 & $1.33 \pm 0.11$ & $1.306 \pm 0.022$ & $0.266 \pm 0.014$ & 9 \\
\hline HD 20794 & G8 V & $6.04 \pm 0.01$ & 0.663 & 5413 & -0.34 & 6.22 & 10.4 & 3 & $0.0148 \pm 0.0018$ & $0.3498 \pm 0.0058$ & 0 & 10 \\
\hline$\epsilon$ Eri & $\mathrm{K} 2 \mathrm{~V}$ & $3.22 \pm 0.01$ & 0.425 & 5061 & -0.08 & 0.8 & $\ldots$ & 1 & $1.05 \pm 0.19$ & $3.38 \pm 0.32$ & $0.25 \pm 0.23$ & 11 \\
\hline HD 33564 & F6 V & $20.88 \pm 0.09$ & 3.224 & 6307 & 0.10 & 5.7 & 1.2 & 1 & $9.13 \pm 0.37$ & $1.124 \pm 0.020$ & $0.34 \pm 0.02$ & 12 \\
\hline HD 39091 & G1 IV & $18.32 \pm 0.07$ & 1.535 & 6003 & 0.09 & 5.1 & 3.2 & 1 & $10.09 \pm 0.38$ & $3.35 \pm 0.10$ & $0.6405 \pm 0.0072$ & 13 \\
\hline HD 40307 & K3 V & $13.00 \pm 0.06$ & 0.245 & 4979 & -0.31 & 7.3 & 6.7 & 3 & $0.0281 \pm 0.0002$ & $0.1324 \pm 0.0022$ & 0 & 14 \\
\hline HD 69830 & K0 V & $12.49 \pm 0.05$ & 0.595 & 5405 & -0.04 & 6.4 & 3.3 & 3 & $0.0563 \pm 0.0052$ & $0.627 \pm 0.012$ & $0.10 \pm 0.04$ & 15 \\
\hline $55 \mathrm{Cnc}$ & G8 V & $12.34 \pm 0.11$ & 0.602 & 5295 & 0.36 & 8.4 & 11.2 & 5 & $3.835 \pm 0.08$ & $5.475 \pm 0.094$ & $0.004 \pm 0.003$ & 4 \\
\hline $47 \mathrm{UMa}$ & G0 V & $14.06 \pm 0.05$ & 1.600 & 5908 & 0.03 & 4.9 & & 3 & $2.546 \pm 0.096$ & $3.570 \pm 0.111$ & $0.032 \pm 0.014$ & 16 \\
\hline HD 99492 & $\mathrm{~K} 2 \mathrm{~V}$ & $17.96 \pm 0.46$ & 0.327 & 4810 & 0.26 & 4.0 & 13.0 & 2 & $0.36 \pm 0.02$ & $5.4 \pm 0.1$ & $0.254 \pm 0.092$ & 17 \\
\hline HD 102365 & G3/G5 V & $9.22 \pm 0.02$ & 0.839 & 5630 & -0.28 & 5.7 & 8.83 & 1 & $0.051 \pm 0.008$ & $0.4633 \pm 0.0078$ & $0.34 \pm 0.14$ & 18 \\
\hline $61 \mathrm{Vir}$ & G5 V & $8.56 \pm 0.02$ & 0.835 & 5646 & -0.02 & 6.6 & 8.0 & 3 & $0.0716 \pm 0.0093$ & $0.475 \pm 0.008$ & $0.12 \pm 0.11$ & 19 \\
\hline 70 Vir & G5 V & $17.98 \pm 0.08$ & 2.989 & 5513 & -0.07 & 7.9 & 5.6 & 1 & $7.46 \pm 0.25$ & $0.4836 \pm 0.0081$ & $0.4007 \pm 0.0035$ & 20 \\
\hline$\tau$ Boo & F7 V & $15.62 \pm 0.05$ & 3.062 & 6376 & 0.26 & 4.8 & 0.4 & 1 & $5.95 \pm 0.28$ & 0.046 & $0.023 \pm 0.015$ & 4 \\
\hline$\alpha$ Cen B & $\mathrm{K} 1 \mathrm{~V}$ & $1.25 \pm 0.04$ & 0.444 & 5178 & 0.15 & 5.2 & 4.27 & 1 & $0.00356 \pm 0.00028$ & $0.0419 \pm 0.0007$ & 0 & 21 \\
\hline GJ 581 & M5 V & $6.27 \pm 0.09$ & 0.012 & 3315 & -0.02 & 2.00 & 7.0 & $4^{e}$ & $0.0499 \pm 0.0023$ & $0.2177 \pm 0.0047$ & $0.32 \pm 0.09$ & 22 \\
\hline$\rho \mathrm{CrB}$ & $\mathrm{G} 2 \mathrm{~V}$ & $17.24 \pm 0.08$ & 1.742 & 5834 & -0.19 & 4.3 & $<1.09$ & 1 & $1.064 \pm 0.053$ & $0.2257 \pm 0.0038$ & $0.057 \pm 0.028$ & 23 \\
\hline 14 Her & K0 V & $17.57 \pm 0.10$ & 0.653 & 5336 & 0.43 & 6.9 & 7.5 & 1 & $5.21 \pm 0.30$ & $2.934 \pm 0.084$ & $0.369 \pm 0.005$ & 24 \\
\hline HD 154345 & G8 V & $18.58 \pm 0.11$ & 0.617 & 5488 & -0.07 & 3.8 & 5.6 & 1 & $0.957 \pm 0.061$ & $4.21 \pm 0.11$ & $0.044_{-0.044}^{+0.046}$ & 25 \\
\hline$\mu$ Ara & G5 V & $15.51 \pm 0.07$ & 1.821 & 5787 & 0.29 & 7.5 & $\ldots$ & 4 & $1.89 \pm 0.22$ & $5.34 \pm 0.40$ & $0.172 \pm 0.040$ & 26 \\
\hline HD 176051 & G0 V & $14.87 \pm 0.08$ & 1.603 & 5840 & -0.11 & 8.1 & 1.1 & 1 & $1.50 \pm 0.30$ & $1.76 \pm 0.07$ & 0 & 27 \\
\hline 16 Cyg B & G5 V & $21.21 \pm 0.12$ & 1.271 & 5772 & 0.08 & 7.4 & $<10.3$ & 1 & $1.640 \pm 0.083$ & $1.660 \pm 0.028$ & $0.681 \pm 0.017$ & 28 \\
\hline HD 189567 & $\mathrm{G} 2 \mathrm{~V}$ & $17.73 \pm 0.14$ & 1.027 & 5735 & -0.22 & 4.1 & $\ldots$ & 1 & $0.0316 \pm 0.0034$ & $0.1099 \pm 0.0018$ & $0.23 \pm 0.14$ & 29 \\
\hline HD 192310 & K3 V & $8.91 \pm 0.02$ & 0.407 & 5105 & -0.03 & 7.5 & 2.9 & 2 & $0.0736 \pm 0.0098$ & $1.184 \pm 0.024$ & $0.13 \pm 0.04$ & 30 \\
\hline GJ 832 & M1 V & $7.86 \pm 0.12$ & 0.031 & 3695 & -0.31 & $\ldots$ & 9.2 & 1 & $0.644 \pm 0.075$ & $3.40 \pm 0.15$ & $0.12 \pm 0.11$ & 31 \\
\hline HD 210277 & G0 V & $21.56 \pm 0.22$ & 1.002 & 5540 & 0.22 & 6.8 & $\ldots$ & 1 & $1.273 \pm 0.005$ & $1.131 \pm 0.019$ & $0.476 \pm 0.017$ & 32 \\
\hline GJ 876 & M5 V & $4.69 \pm 0.05$ & 0.013 & 3473 & 0.19 & 6.5 & $\ldots$ & 4 & $2.2756 \pm 0.0045$ & $0.3343 \pm 0.0013$ & $0.207 \pm 0.055$ & 33 \\
\hline $51 \mathrm{Peg}$ & G5 V & $15.61 \pm 0.09$ & 1.368 & 5791 & 0.20 & 8.0 & 13.6 & 1 & $0.461 \pm 0.016$ & $0.05211 \pm 0.00087$ & $0.013 \pm 0.012$ & 34 \\
\hline HD 217107 & G8 IV & $19.86 \pm 0.15$ & 1.162 & 5646 & 0.4 & 8.1 & $\ldots$ & 2 & $2.62 \pm 0.15$ & $5.33 \pm 0.02$ & $0.1276 \pm 0.0052$ & 35 \\
\hline
\end{tabular}

Notes. ${ }^{(a)}$ Number of known exoplanets. ${ }^{(b)}$ Projected (minimum) mass of the most massive exoplanet. ${ }^{(c)}$ Semi-major axis of the outermost exoplanet in the system. ${ }^{(d)}$ Eccentricity of innermost exoplanet in the system. ${ }^{(e)}$ Vogt et al. (2012) reported strong evidence for a five-planet system. A summary of all reported exoplanets in each system is given in Table 4. Planets for which no eccentricity could be found are assumed to have $e=0.00$.

References. Discovery references: (1) Naef et al. (2001); (2) Fischer et al. (2003); (3) Udry et al. (2006); (4) Butler et al. (1997); (5) Butler et al. (2006); (6) Tuomi et al. (2013); (7) Queloz et al. (2000); (8) Kürster et al. (2000); (9) Mayor et al. (2004); (10) Pepe et al. (2011); (11) Hatzes et al. (2000); (12) Galland et al. (2005); (13) Jones et al. (2002); (14) Mayor et al. (2009); (15) Lovis et al. (2006); (16) Butler \& Marcy (1996); (17) Marcy et al. (2005); (18) Tinney et al. (2011); (19) Vogt et al. (2010); (20) Marcy \& Butler (1996); (21) Dumusque et al. (2012); (22) Bonfils et al. (2005); (23) Noyes et al. (1997); (24) Butler et al. (2003); (25) Wright et al. (2007); (26) Butler et al. (2001); (27) Muterspaugh et al. (2010); (28) Cochran et al. (1997); (29) Mayor et al. (2011); (30) Howard et al. (2011); (31) Bailey et al. (2009); (32) Marcy et al. (1999); (33) Delfosse et al. (1998); (34) Mayor \& Queloz (1995); (35) Fischer et al. (1999). 
Table 4. Summary table of all exoplanets in each system.

\begin{tabular}{|c|c|c|c|c|c|c|}
\hline \multirow[t]{2}{*}{ Name } & \multirow[t]{2}{*}{ Planet } & \multirow{2}{*}{$\begin{array}{c}\operatorname{Mass}(M \sin i) \\
{\left[M_{\mathrm{Jup}}\right]} \\
\end{array}$} & \multirow{2}{*}{$\begin{array}{c}\text { Semi-major axis } \\
{[\mathrm{AU}]}\end{array}$} & \multirow[t]{2}{*}{ Eccentricity } & \multicolumn{2}{|c|}{ Reference } \\
\hline & & & & & Orbit & Discovery \\
\hline HD 1237 & $\mathrm{~b}$ & $3.37 \pm 0.09$ & $0.4947 \pm 0.0083$ & $0.511 \pm 0.017$ & 1 & 1 \\
\hline HD 3651 & $\mathrm{~b}$ & $0.229 \pm 0.0148$ & $0.2947 \pm 0.0049$ & $0.596 \pm 0.036$ & 2 & 3 \\
\hline HD 4308 & $\mathrm{~b}$ & $0.0477 \pm 0.0028$ & $0.1192 \pm 0.00199$ & $0.000 \pm{ }_{-0.00}^{0.01}$ & 4 & 4 \\
\hline \multirow{3}{*}{$v$ And } & $\mathrm{b}$ & $0.669 \pm 0.026$ & $0.05939 \pm 0.00099$ & $0.013 \pm{ }_{0.013}^{0.816}$ & 5 & 6 \\
\hline & $\mathrm{c}$ & $1.919 \pm 0.088$ & $0.830 \pm 0.0138$ & $0.224 \pm 0.026$ & 5 & 7 \\
\hline & $\mathrm{d}$ & $4.12 \pm 0.165$ & $2.525 \pm 0.042$ & $0.267 \pm 0.0196$ & 5 & 7 \\
\hline$q^{1}$ Eri & $\mathrm{b}$ & $0.93 \pm 0.24$ & $2.022 \pm 0.082$ & $0.16 \pm{ }_{0.16}^{0.22}$ & 8 & 8 \\
\hline \multirow[t]{5}{*}{$\tau$ Ceti } & $\mathrm{b}$ & $0.0063 \pm 0.0025$ & $0.105 \pm 0.005$ & $0.16 \pm_{0.16}^{0.22}$ & 9 & 9 \\
\hline & $\mathrm{c}$ & $0.0098 \pm_{0.0035}^{0.0044}$ & $0.195 \pm 0.009$ & $0.03 \pm_{0.03}^{0.28}$ & 9 & 9 \\
\hline & $\mathrm{d}$ & $0.0113 \pm 0.0054$ & $0.374 \pm 0.020$ & $0.08 \pm_{0.0}^{0.2}$ & 9 & 9 \\
\hline & $\mathrm{e}$ & $0.0135 \pm 0.0066$ & $0.552 \pm 0.023$ & $0.05 \pm_{0}^{0}$ & 9 & 9 \\
\hline & $\mathrm{f}$ & $0.0208 \pm 0.0110$ & $1.350 \pm 0.080$ & $0.03 \pm_{0.03}^{0.26}$ & 9 & 9 \\
\hline GJ 86 & $\mathrm{~b}$ & $4.00 \pm 0.137$ & $0.1142 \pm 0.0019$ & $0.0416 \pm 0.0072$ & 8 & 10 \\
\hline$\iota$ Hor & $\mathrm{b}$ & $2.05 \pm 0.2$ & $0.924 \pm 0.0161$ & $0.14 \pm 0.13$ & 8 & 11 \\
\hline HD 19994 & $\mathrm{~b}$ & $1.33 \pm 0.105$ & $1.306 \pm 0.022$ & $0.266 \pm 0.014$ & 2 & 12 \\
\hline \multirow[t]{3}{*}{ HD 20794} & $\mathrm{~b}$ & $0.00849 \pm 0.00096$ & $0.1207 \pm 0.002$ & 0 & 13 & 13 \\
\hline & $\mathrm{c}$ & $0.0074 \pm 0.00135$ & $0.2036 \pm 0.0034$ & 0 & 13 & 13 \\
\hline & $\mathrm{d}$ & $0.0148 \pm 0.00181$ & $0.3498 \pm 0.0058$ & 0 & 13 & 13 \\
\hline$\epsilon$ Eri & b & $1.05 \pm 0.188$ & $3.38 \pm 0.32$ & $0.25 \pm 0.23$ & 8 & 14 \\
\hline HD 33564 & $\mathrm{~b}$ & $9.13 \pm 0.37$ & $1.124 \pm 0.0196$ & $0.340 \pm 0.02$ & 15 & 15 \\
\hline HD 39091 & $\mathrm{~b}$ & $10.09 \pm 0.38$ & $3.35 \pm 0.104$ & $0.6405 \pm 0.0072$ & 8 & 16 \\
\hline \multirow{3}{*}{ HD 40307} & b & $0.01291 \pm 0.00084$ & $0.04689 \pm 0.00078$ & 0 & 17 & 17 \\
\hline & $\mathrm{c}$ & $0.0211 \pm 0.00118$ & $0.0801 \pm 0.00133$ & 0 & 17 & 17 \\
\hline & $\mathrm{d}$ & $0.0281 \pm 0.00162$ & $0.1324 \pm 0.0022$ & 0 & 17 & 17 \\
\hline \multirow[t]{3}{*}{ HD 69830} & $\mathrm{~b}$ & $0.0316 \pm 0.00172$ & $0.0782 \pm 0.0013$ & $0.100 \pm 0.04$ & 18 & 18 \\
\hline & c & $0.0368 \pm 0.0025$ & $0.1851 \pm 0.0031$ & $0.130 \pm 0.06$ & 18 & 18 \\
\hline & $\mathrm{d}$ & $0.0563 \pm 0.0052$ & $0.627 \pm 0.0122$ & $0.070 \pm 0.07$ & 18 & 18 \\
\hline \multirow[t]{5}{*}{$55 \mathrm{Cnc}$} & $\mathrm{b}$ & $0.801 \pm 0.027$ & $0.1134 \pm 0.00189$ & $0.0040 \pm 0.003$ & 19 & 6 \\
\hline & $\mathrm{c}$ & $0.1646 \pm 0.0066$ & $0.2373 \pm 0.004$ & $0.070 \pm 0.02$ & 19 & 20 \\
\hline & $\mathrm{d}$ & $3.54 \pm 0.122$ & $5.475 \pm 0.094$ & $0.0200 \pm 0.008$ & 19 & 20 \\
\hline & e & $0.0262 \pm 0.00123$ & $0.01544 \pm 0.00026$ & 0 & 19 & 21 \\
\hline & $\mathrm{f}$ & $0.173 \pm 0.0106$ & $0.774 \pm 0.0129$ & $0.320 \pm 0.05$ & 19 & 22 \\
\hline \multirow[t]{2}{*}{$47 \mathrm{UMa}$} & $\mathrm{b}$ & $2.546 \pm 0.096$ & $2.101 \pm 0.035$ & $0.032 \pm 0.014$ & 23 & 24 \\
\hline & $\mathrm{c}$ & $0.546 \pm 0.071$ & $3.57 \pm 0.111$ & $0.098 \pm 0.071$ & 23 & 25 \\
\hline \multirow[t]{2}{*}{ HD 99492} & $\mathrm{~b}$ & $0.106 \pm 0.0117$ & $0.1219 \pm 0.002$ & $0.254 \pm 0.092$ & 8 & 26 \\
\hline & c & $0.36 \pm 0.02$ & $5.4 \pm 0.1$ & $0.106 \pm 0.006$ & 27 & 27 \\
\hline HD 102365 & $\mathrm{~b}$ & $0.0510 \pm 0.0081$ & $0.4633 \pm 0.0078$ & $0.34 \pm 0.14$ & 28 & 28 \\
\hline \multirow{3}{*}{$61 \mathrm{Vir}$} & $\mathrm{b}$ & $0.0161 \pm 0.00184$ & $0.05006 \pm 0.00083$ & $0.12 \pm 0.11$ & 29 & 29 \\
\hline & $\mathrm{c}$ & $0.0334 \pm 0.0038$ & $0.2169 \pm 0.0036$ & $0.140 \pm 0.06$ & 29 & 29 \\
\hline & $\mathrm{d}$ & $0.0716 \pm 0.0093$ & $0.4745 \pm 0.008$ & $0.350 \pm 0.09$ & 29 & 29 \\
\hline 70 Vir & $\mathrm{b}$ & $7.46 \pm 0.25$ & $0.4836 \pm 0.0081$ & $0.4007 \pm 0.0035$ & 8 & 30 \\
\hline$\tau$ Boo & $\mathrm{b}$ & $4.17 \pm 0.142$ & $0.04800 \pm 0.0008$ & $0.023 \pm 0.015$ & 31 & 6 \\
\hline$\alpha$ Cen B & $\mathrm{b}$ & $0.00356 \pm 0.00028$ & $0.0419 \pm 0.0007$ & 0 & 32 & 32 \\
\hline \multirow[t]{4}{*}{ GJ 581} & $\mathrm{~b}$ & $0.0499 \pm 0.0023$ & $0.04061 \pm 0.00087$ & $0.031 \pm 0.014$ & 33 & 34 \\
\hline & $\mathrm{c}$ & $0.0168 \pm 0.00119$ & $0.0729 \pm 0.00157$ & $0.070 \pm 0.06$ & 33 & 35 \\
\hline & $\mathrm{d}$ & $0.0191 \pm 0.0022$ & $0.2177 \pm 0.0047$ & $0.250 \pm 0.09$ & 33 & 35 \\
\hline & $\mathrm{e}$ & $0.00613 \pm 0.00071$ & $0.02846 \pm 0.00061$ & $0.320 \pm 0.09$ & 33 & 17 \\
\hline$\rho \mathrm{CrB}$ & $\mathrm{b}$ & $1.064 \pm 0.053$ & $0.2257 \pm 0.0038$ & $0.057 \pm 0.028$ & 8 & 36 \\
\hline 14 Her & $\mathrm{b}$ & $5.21 \pm 0.3$ & $2.934 \pm 0.084$ & $0.3690 \pm 0.005$ & 37 & 38 \\
\hline HD 154345 & $\mathrm{~b}$ & $0.957 \pm 0.061$ & $4.21 \pm 0.105$ & $0.044 \pm \begin{array}{l}0.046 \\
0.044\end{array}$ & 39 & 40 \\
\hline \multirow{3}{*}{$\mu$ Ara } & $\mathrm{b}$ & $1.746 \pm 0.069$ & $1.527 \pm 0.029$ & $0.128 \pm 0.017$ & 41 & 42 \\
\hline & $\mathrm{c}$ & $1.89 \pm 0.22$ & $5.34 \pm 0.4$ & $0.099 \pm 0.063$ & 41 & 43 \\
\hline & $\mathrm{d}$ & $0.0346 \pm 0.00199$ & $0.0928 \pm 0.00177$ & $0.172 \pm 0.04$ & 41 & 44 \\
\hline
\end{tabular}

References. (1) Naef et al. (2001); (2) Wittenmyer et al. (2009); (3) Fischer et al. (2003); (4) Udry et al. (2006); (5) Wright et al. (2009); (6) Butler et al. (1997); (7) Butler et al. (1999); (8) Butler et al. (2006); (9) Tuomi et al. (2013); (10) Queloz et al. (2000); (11) Kürster et al. (2000); (12) Mayor et al. (2004); (13) Pepe et al. (2011); (14) Hatzes et al. (2000); (15) Galland et al. (2005); (16) Jones et al. (2002); (17) Mayor et al. (2009); (18) Lovis et al. (2006); (19) Endl et al. (2012); (20) Marcy et al. (2002); (21) McArthur et al. (2004); (22) Fischer et al. (2008); (23) Gregory \& Fischer (2010); (24) Butler \& Marcy (1996); (25) Fischer et al. (2002); (26) Marcy et al. (2005); (27) Meschiari et al. (2011); (28) Tinney et al. (2011); (29) Vogt et al. (2010); (30) Marcy \& Butler (1996); (31) Brogi et al. (2012); (32) Dumusque et al. (2012); (33) Forveille et al. (2011); (34) Bonfils et al. (2005); (35) Udry et al. (2007); (36) Noyes et al. (1997); (37) Wittenmyer et al. (2007); (38) Butler et al. (2003); (39) Wright et al. (2008); (40) Wright et al. (2007); (41) Pepe et al. (2007); (42) Butler et al. (2001); (43) McCarthy et al. (2004); (44) Santos et al. (2004); (45) Goździewski et al. (2007); (46) Muterspaugh et al. (2010); (47) Cochran et al. (1997); (48) Mayor et al. (2011); (49) Howard et al. (2011); (50) Bailey et al. (2009); (51) Marcy et al. (1999); (52) Rivera et al. (2010); (53) Delfosse et al. (1998); (54) Marcy et al. (1998); (55) Marcy et al. (2001); (56) Rivera et al. (2005); (57) Mayor \& Queloz (1995); (58) Fischer et al. (1999); (59) Vogt et al. (2005). 
Table 4. continued.

\begin{tabular}{llccccc}
\hline \hline Name & Planet & $\begin{array}{c}\text { Mass }(M \sin i) \\
{\left[M_{\text {Jup }}\right]}\end{array}$ & $\begin{array}{c}\text { Semi-major axis } \\
{[\mathrm{AU}]}\end{array}$ & Eccentricity & \multicolumn{2}{c}{ Reference } \\
& & $0.543 \pm 0.03$ & $0.940 \pm 0.018$ & $0.067 \pm 0.0122$ & 41 & 41,45 \\
& $\mathrm{e}$ & $1.5 \pm 0.3$ & 19.1 & $0.2667 \pm 0.0022$ & 46 & 46 \\
HD 176051 & $\mathrm{b}$ & $1.640 \pm 0.083$ & $1.660 \pm 0.028$ & $0.681 \pm 0.017$ & 8 & 47 \\
16 Cyg B & $\mathrm{b}$ & $0.0316 \pm 0.0034$ & $0.1099 \pm 0.0018$ & $0.23 \pm 0.14$ & 48 & 48 \\
HD 189567 & $\mathrm{b}$ & $0.3223 \pm 0.0054$ & $0.130 \pm 0.04$ & 13 & 49 \\
HD 192310 & $\mathrm{b}$ & $0.0531 \pm 0.0028$ & 0.13 & 13 \\
& $\mathrm{c}$ & $0.0736 \pm 0.0098$ & $1.184 \pm 0.024$ & $0.32 \pm 0.11$ & 13 & 13 \\
GJ 832 & $\mathrm{b}$ & $0.644 \pm 0.075$ & $3.40 \pm 0.153$ & $0.12 \pm 0.11$ & 50 & 50 \\
HD 210277 & $\mathrm{b}$ & $1.273 \pm 0.051$ & $1.131 \pm 0.0189$ & $0.476 \pm 0.017$ & 8 & 51 \\
GJ 876 & $\mathrm{b}$ & $1.95 \pm 0.122$ & $0.2081 \pm 0.0065$ & $0.0324 \pm 0.0013$ & 52 & 53,54 \\
& $\mathrm{c}$ & $0.612 \pm 0.021$ & $0.1296 \pm 0.0022$ & $0.25591 \pm 0.00093$ & 52 & 55 \\
& $\mathrm{~d}$ & $0.0184 \pm 0.00123$ & $0.02081 \pm 0.00035$ & $0.207 \pm 0.055$ & 52 & 56 \\
51 Peg & $\mathrm{e}$ & $0.0392 \pm 0.0051$ & $0.333 \pm 0.0105$ & $0.055 \pm 0.012$ & 52 & 52 \\
HD 217107 & $\mathrm{b}$ & $0.461 \pm 0.0164$ & $0.05211 \pm 0.00087$ & $0.013 \pm 0.012$ & 8 & 57 \\
& $\mathrm{c}$ & $1.401 \pm 0.048$ & $0.0750 \pm 0.00125$ & $0.1267 \pm 0.0052$ & 5 & 58 \\
\hline
\end{tabular}

